\title{
EchoGéo
}

$51 \mid 2020$

Territoires « cyclonés »

\section{Apports de l'imagerie satellite pour le suivi de l'impact des événements cycloniques à Madagascar}

Complémentarité des échelles et des capteurs

Thibault Catry, Christophe Révillion, Pascal Mouquet et Gwenaëlle Pennober

\section{QpenEdition}

\section{Journals}

Édition électronique

URL : https://journals.openedition.org/echogeo/18634

DOI : 10.4000/echogeo.18634

ISSN : 1963-1197

Éditeur

Pôle de recherche pour l'organisation et la diffusion de l'information géographique (CNRS UMR 8586)

Référence électronique

Thibault Catry, Christophe Révillion, Pascal Mouquet et Gwenaëlle Pennober, « Apports de l'imagerie satellite pour le suivi de l'impact des événements cycloniques à Madagascar », EchoGéo [En ligne], 51 | 2020, mis en ligne le 25 avril 2020, consulté le 11 août 2021. URL : http://journals.openedition.org/ echogeo/18634; DOI : https://doi.org/10.4000/echogeo.18634

Ce document a été généré automatiquement le 11 août 2021.

EchoGéo est mis à disposition selon les termes de la licence Creative Commons Attribution - Pas d'Utilisation Commerciale - Pas de Modification 4.0 International (CC BY-NC-ND) 


\title{
Apports de l'imagerie satellite pour le suivi de l'impact des événements cycloniques à Madagascar
}

Complémentarité des échelles et des capteurs

\author{
Thibault Catry, Christophe Révillion, Pascal Mouquet et Gwenaëlle \\ Pennober
}

Cette étude a bénéficié des ressources en données d'observation de la Terre et traitement d'images de la station SEAS-OI de La Réunion et du programme Copernicus de l'ESA. Certains travaux ont été réalisés dans le cadre du projet FEDER OBSAT (2012-2015) et valorisés dans le contexte du projet INTERREG Renovrisk-Impact (2018-2021).

\section{Contexte et zones d'étude}

1 L'île-continent Madagascar, de par sa position dans le sud-ouest de l'océan Indien est particulièrement soumise à l'activité cyclonique. Le centre de Météo-France (MF) basé à la Réunion a été désigné par la World Meteorological Organization (WMO), comme l'un des 6 centres mondiaux de surveillance des cyclones (CMRS, Centre Météorologique Régional Spécialisé/Cyclones), en charge de la surveillance des systèmes cycloniques du sud-ouest de l'océan Indien (entre l'Afrique et $90^{\circ} \mathrm{E}$ ). D'après la classification des principaux systèmes dépressionnaires tropicaux utilisée dans le sud-ouest de l'océan Indien définie par MF, le stade de cyclone tropical est atteint lorsque la valeur maximale de la vitesse moyenne du vent est supérieure à 64 nœuds $(118 \mathrm{~km} / \mathrm{h}$, force ouragan 12 Beaufort).

2 Entre 1985 et 2015, 306 systèmes dépressionnaires ont été répertoriés dans ce bassin océanique (une moyenne de neuf par saison cyclonique) dont 154 cyclones (quatre par saison en moyenne avec une variabilité forte selon les années: huit systèmes en 2002-2003 et 2006-2007 contre seulement un en 2010-2011 et 2015-2016). 45 cyclones et tempêtes tropicales ont été répertoriés au cours des dix dernières années (illustration 1). 
Illustration 1 - Nombre de systèmes dépressionnaires répertoriés dans l'océan Indien depuis la saison 1984-1985, avec en orange les cyclones

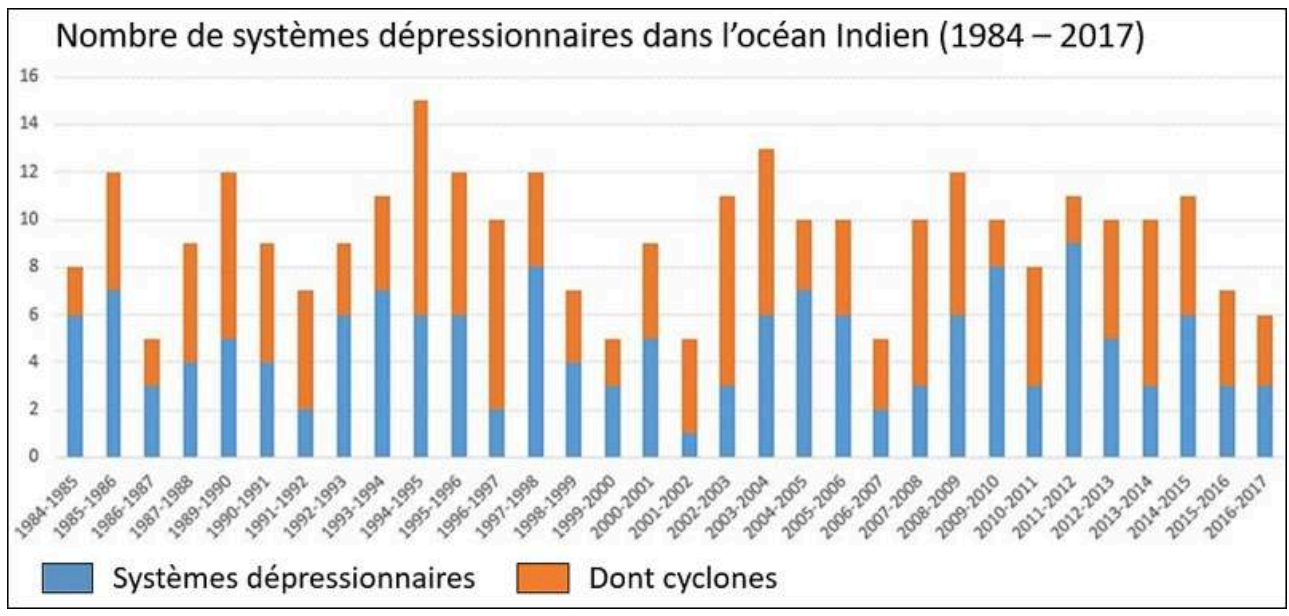

Source : Met Office, JTWC.

Les données best tracks du JTWC (Joint Typhoon Warning Center de l'agence américaine Department of Defense), nous ont permis d'établir une cartographie des zones les plus touchées par les cyclones (vents moyens $\geq 64$ noeuds) depuis 1980 (illustration 2). Cette carte a été établie en compilant 9333 points de trajectoires de cyclones constituant 475 traces de l'est de l'hémisphère sud (océans Indien et Pacifique). Les valeurs représentent le nombre de cyclones dont le cœur est passé à une distance inférieure à $100 \mathrm{~km}$. On note une très forte hétérogénéité sur la zone du sud-ouest de l'océan Indien (SWOI), avec des valeurs s'échelonnant entre 0 et plus de 17 cyclones. Les trajectoires forment de véritables couloirs de passages, notamment entre le $15^{\mathrm{e}}$ et le $20^{\mathrm{e}}$ degré de latitude sud. La zone du Canal du Mozambique (maximum 7 cyclones) est moins touchée que l'est de Madagascar. 
Illustration 2 - Distribution spatiale des cyclones dans le SWOI entre 1980 et 2017

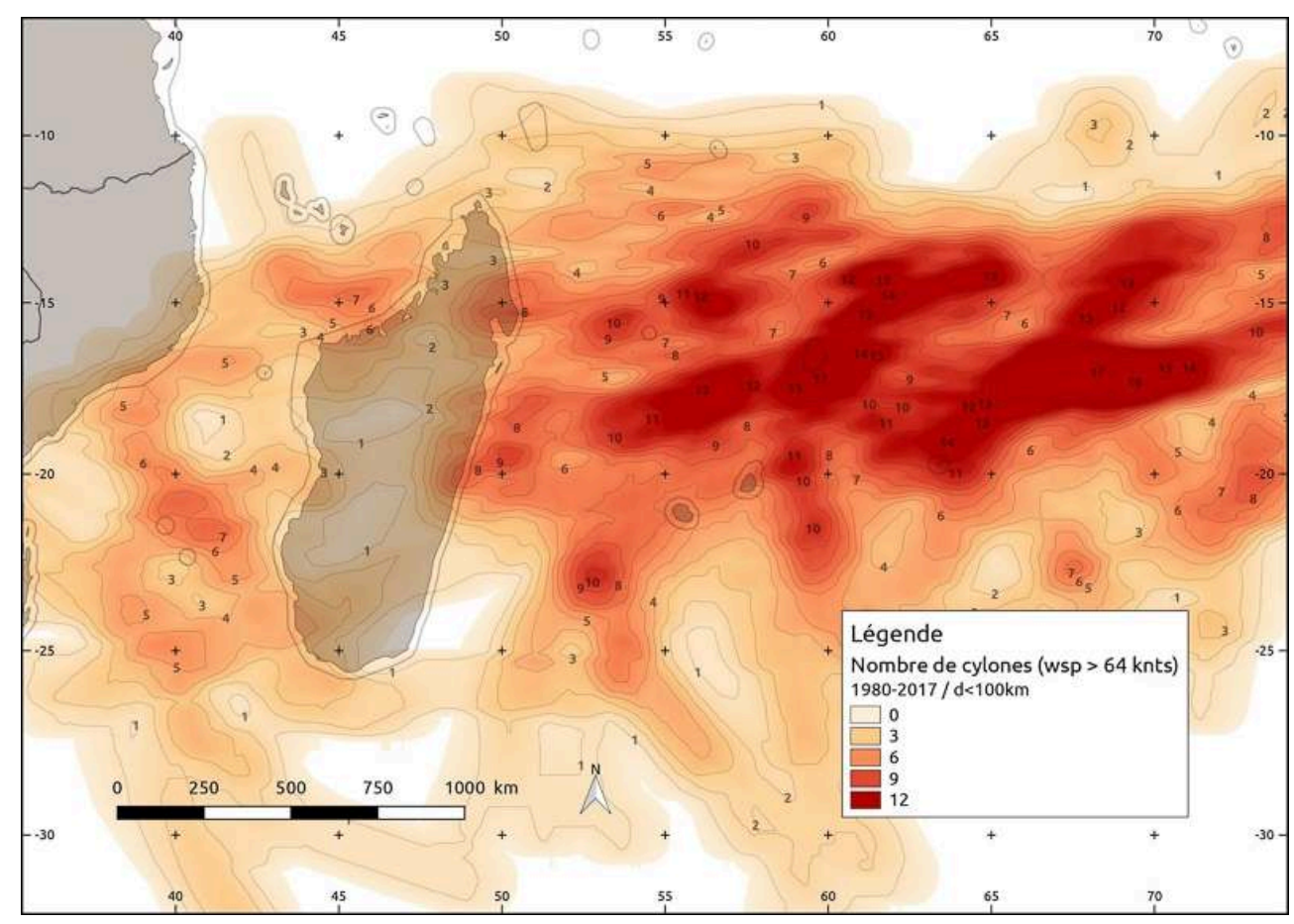

Source : best tracks du JTWC. Auteur: P. Mouquet.

4 En raison de son étirement en latitude sur près de 15 degrés entre le $12^{\mathrm{e}}$ et le $26^{\mathrm{e}}$ degré sud, l'influence cyclonique n'est pas homogène sur l'ensemble de Madagascar, avec entre 0 et 8 systèmes qui ont touché lîlle sur la période. Les zones les plus touchées se trouvent sur les côtes, au nord et à l'est de l'île (provinces de Diego-Suarez, Majunga et Tamatave). On peut noter plusieurs hotspots :

- au nord-est: la presqu'île de Masoala, la Baie d'Antongil jusqu'à la ville de Sambava vers le Nord, avec entre six et huit cyclones constitue une des zones plus touchées. Les villes principales de la zone sont : Antalaha, Sambava, Maroantsetra;

- Aàl'est, la région d'Atsinanana dans la province de Tamatave entre cinq et sept cyclones. La principale ville de la zone est Tamatave ;

- au nord-ouest, la région de Boeny dans la province de Mahajanga est aussi une zone d'activité cyclonique importante (six systèmes). La principale ville de la région est Mahajanga ;

- si le sud de l'île est plus épargné, on peut noter que la zone de Toliara au sud-ouest est un peu plus touchée ( $>4$ systèmes).

5 Madagascar figurant parmi les pays les plus pauvres au monde (PIB/habitant en 2016 : 391 USD $\left.^{1}\right)$, l'impact des cyclones se traduit par d'importantes pertes en vies humaines et une augmentation de la fragilité économique (Brown, 2009). Les conséquences en chaîne des cyclones à l'échelle d'un territoire insulaire comme Madagascar sont extrêmement variées et vont largement être conditionnées par leurs intensités et leurs positions par rapport à l'île. Lorsque l'œil du cyclone frappe directement les côtes, les vents, les précipitations, la houle et la surcote sont particulièrement dommageables. Lorsque la trajectoire du cyclone reste en mer à une distance plus éloignée, les conséquences sont moindres mais l'île peut être sous l'influence de la houle et de bandes précipitantes intenses; les effets locaux sont caractérisés par des risques liés 
aux fortes vagues et de crues importantes suivies d'une phase plus ou moins longue de décrue.

Cette étude s'intéresse à deux évènements marquants en termes d'impacts sur les cinq dernières années : (i) le cyclone Haruna, qui a frappé la côte sud-ouest de Madagascar en février 2013, plus particulièrement la province de Toliara (région d'AtsimoAndrefana, illustration 3) et (ii) le cyclone Enawo, qui a touché la côte nord-est de Madagascar au niveau de Sambava (région de Sava, province d'Antsiranana) et Maroantsetra (région d'Analanjirofo, province de Tamatave) en mars 2017 (illustration 3).

Illustration 3 - Localisation des zones d'étude : au sud-ouest l'agglomération de Toliara (province de Toliara), touchée par le cyclone Haruna en 2013 ; au nord Est, Sambava (province d'Antsiranana) et Maroantsetra (province de Tamatave) touchées par Enawo en 2017

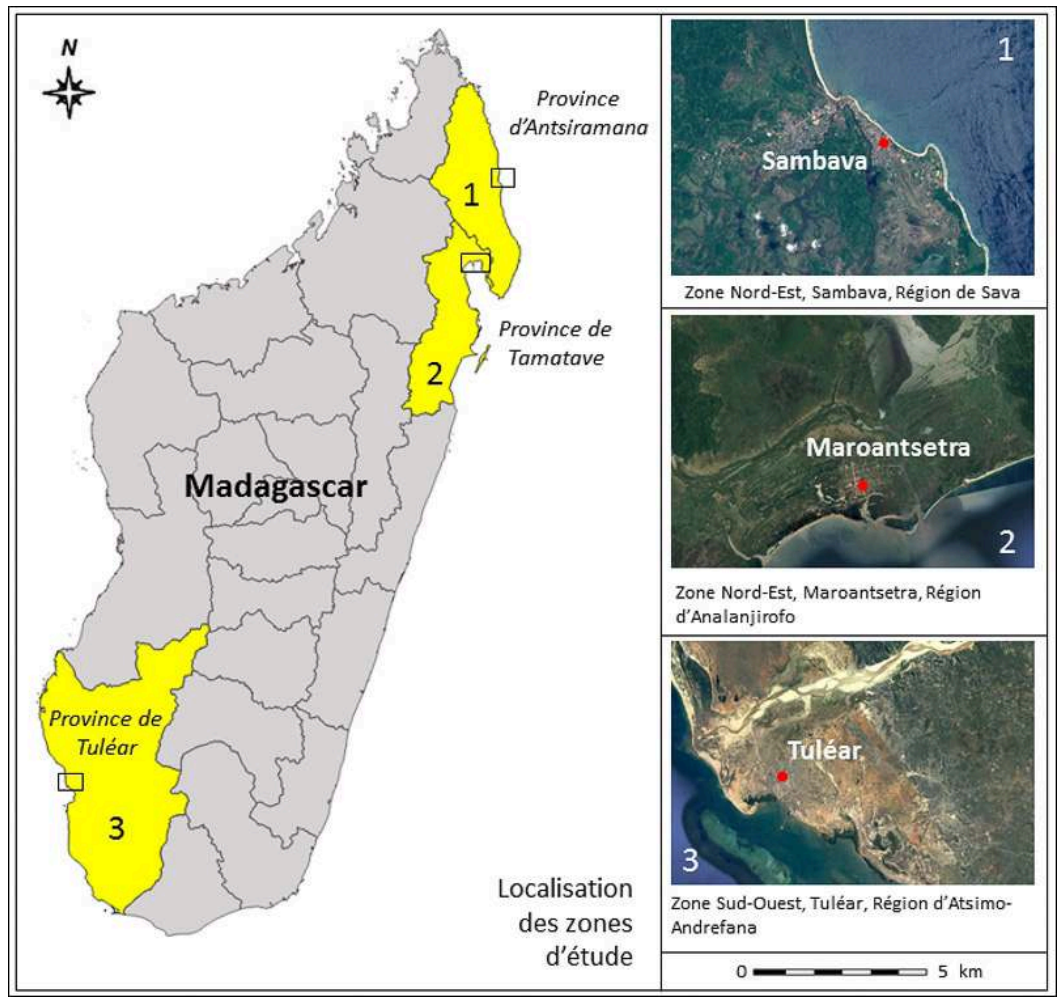

Auteur : T. Catry

7 Les trois sites d'étude sont des villes de plaines alluviales côtières. La dégradation des bassins versants se traduit par une forte sédimentation littorale qui renforce le risque d'inondation par l'obstruction des embouchures et la surélévation des cours d'eau². Toliara, sur la côte sud se développe sur le delta fluviatile du fleuve Fiherenana. La partie la plus haute de la ville se situe à une altitude de $16 \mathrm{~m}$, alors que le centre-ville est situé à $10 \mathrm{~m}$ d'altitude, avec une pente moyenne de 1 degré entre la berge du fleuve et la ville de Toliara. Le fleuve se caractérise par un régime intermittent: de mars à novembre il peut présenter un régime endoréique en raison de la péjoration de pluies et de l'augmentation de l'irrigation sur le bassin versant (Chaperon et al. 1993). Cette caractéristique pénalise l'avancée de son delta mais a favorisé l'établissement de la ville portuaire (Salomon, 2009). Les crues du fleuve sont signalées dès lors que le site acquiert le statut de ville le plus souvent après le passage de cyclones. Une digue de $2,5 \mathrm{~km}$ vise à contenir l'écoulement du fleuve au nord de la ville. En 2013, la ville de 
Toliara, comptait environ 157000 habitants $^{3}$, ce qui représente un triplement de la population depuis 1960 favorisé par un fort exode rural. Les deux villes de la côte nordouest sont établies sur et en arrière de cordons littoraux sableux d'embouchure dont la formation a créé des marécages et étangs par les difficultés d'écoulement qu'ils entraînent au fur et à mesure de leur accrétion. La ville de Sambava, sur la côte nordest est installée à l'embouchure formée par la confluence de quatre entités fluviatiles dont le fleuve Bemarivo. En 2015, la ville comptait environ 40000 habitants répartis entre les rives du fleuve et un cordon littoral en front de mer. Maroantsetra, dans la baie d'Antongil, est quant à elle une petite ville portuaire située sur le delta marin du fleuve l'Antalambalana. Elle comptait environ 24000 habitants en 2015. Le profil d'élévation de Maroantsetra, perpendiculairement à la rivière Antenambalana en se dirigeant vers l'ouest montre l'exposition de la ville aux inondations, avec une élévation de seulement 1 à 3 mètres entre la ville et la rivière, et une altitude maximum de 7 à $11 \mathrm{~m}$ dans la ville.

En raison de la diversité des approches et des contextes les deux événements sont présentés ici successivement.

\section{Cas d'étude $\mathrm{n}^{\circ} 1$ : le cyclone Haruna de février 2013, côte sud-ouest de Madagascar}

\section{Observation du cyclone par imagerie basse résolution}

9 La côte sud-ouest de Madagascar, qui borde le canal du Mozambique, a été frappée par le cyclone Haruna en février 2013. Bien que rarement touché par des événements cycloniques majeurs (Jumaux, 2011), ce littoral avait déjà été fortement impacté par le cyclone Bingiza en février 2011, qui avait notamment endommagé la digue protégeant la ville de Toliara du débordement du fleuve Fiherenana (illustration 3). Haruna atteint le stade de cyclone tropical le 20 février, et touche la province de Toliara au stade de forte tempête tropicale le 22 février (illustration 4). Les images du capteur MODIS (satellites Aqua et Terra, NASA) à $1 \mathrm{~km}$ de résolution spatiale d'Haruna les 20 (illustration $4 \mathrm{a}$ ) et 22 (illustration $4 \mathrm{~b}$ ) février permettent de bien suivre le déplacement de la masse nuageuse associée au cyclone. 
Illustration 4 - Images satellites MODIS à $1 \mathrm{~km}$ de résolution spatiale du cyclone Haruna les 20 et 22 février 2013

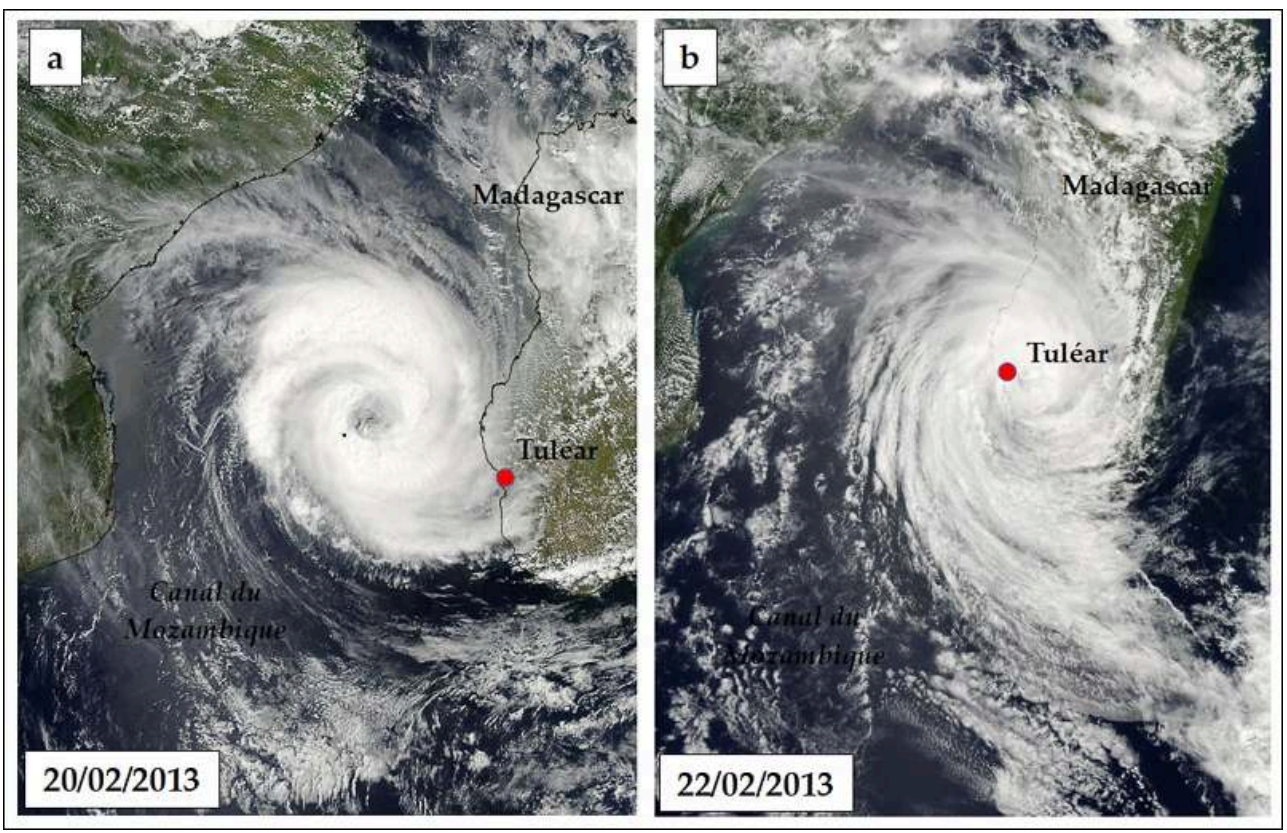

4a : 20/02/2013, Aqua ; 4b, 22/02/2013, Terra

Source: NASA.

10 Les services météorologiques internationaux et le bureau national de Gestion des Risques et des Catastrophes (BNGRC) de Madagascar, ont mis en place une alerte de niveau 4 sur le passage du cyclone Haruna (illustration 5a). Le 19 février, le sud de Madagascar, incluant la province de Toliara, est placé en «alerte orange " (avis de menace; illustration $5 b$ ) puis en "alerte rouge danger imminent» le 20 février (illustration 5c) avec des vents moyens jusqu'à $150 \mathrm{~km} / \mathrm{h}$ et des pics à $210 \mathrm{~km} / \mathrm{h}$. L'alerte est levée le 23 février (illustration 5e). 
Illustration 5 - Trajectoire estimée et observée d'Haruna et niveaux d'alerte émis lors du passage du cyclone par les services météorologiques et le BNGRC de Madagascar
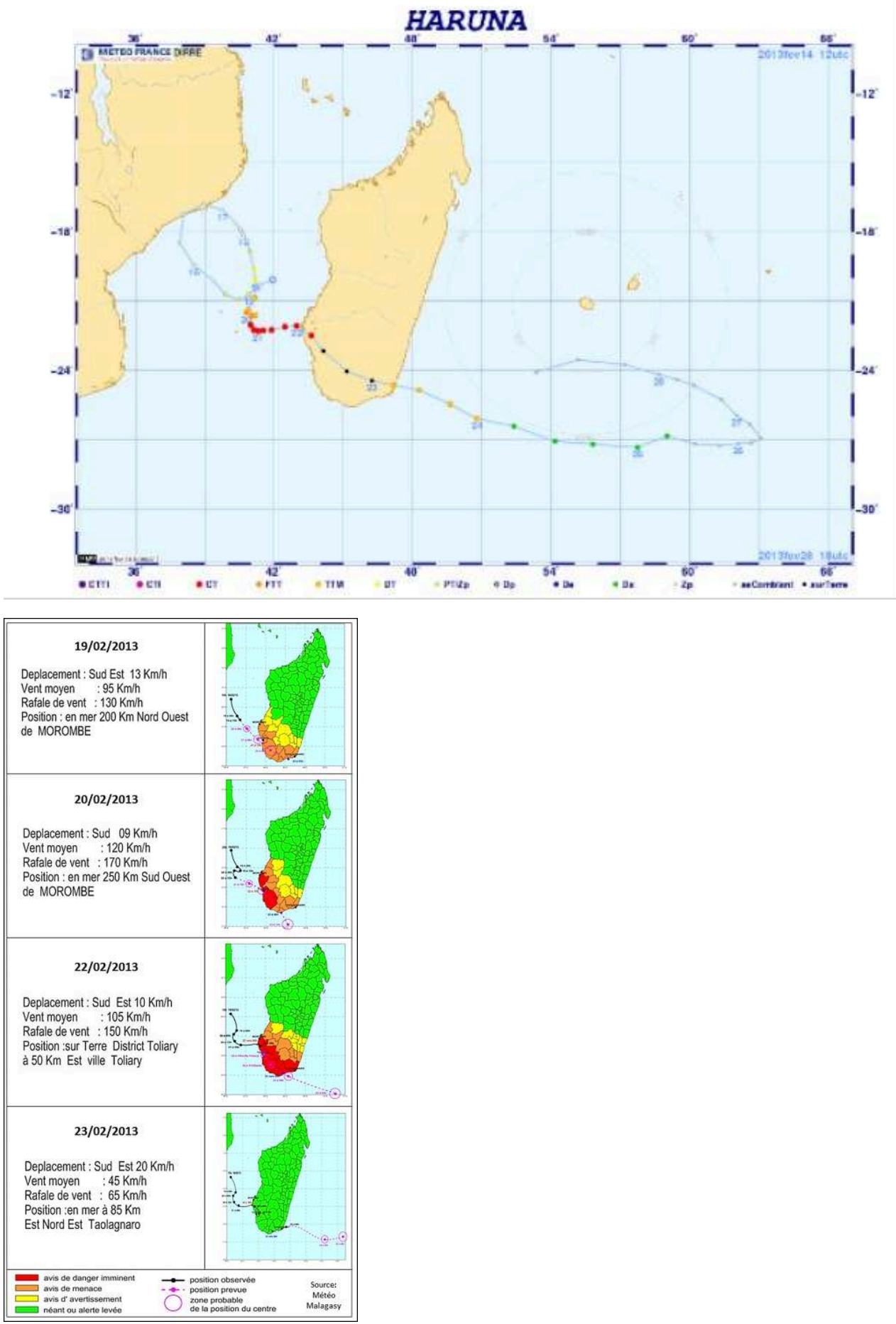

Source des cartes d'alerte : météo Malagasy 


\section{Quantification des précipitations associées à Haruna par imagerie satellite}

Le radar de précipitations de la mission TRMM (Tropical Rainfall Measuring Mission, NASA/NOAA) permet une mesure en temps réel des cumuls de précipitations le long de la trajectoire d'Haruna. Le 20 février, alors qu'Haruna atteint son stade maximal de cyclone tropical, les précipitations sont localement mesurées en mer dans $r$ le canal du Mozambique à leur niveau maximal $(>50 \mathrm{~mm} / \mathrm{h})$, mais le phénomène se situe encore majoritairement loin des côtes, avec un impact limité sur la zone de Toliara (illustration 6a). Le 22 février, lorsqu'il touche Toliara, Haruna est redevenu tempête tropicale intense. Les précipitations maximales diminuent mais restent fortes, atteignant localement sur terre 10 à $20 \mathrm{~mm} / \mathrm{h}$ (illustration $6 \mathrm{~b}$ ), causant les premières inondations sur la zone de Toliara (voir illustration 9)

Illustration 6 - Précipitations associées au cyclone Haruna les 20 (a) et 22 (b) février 2013

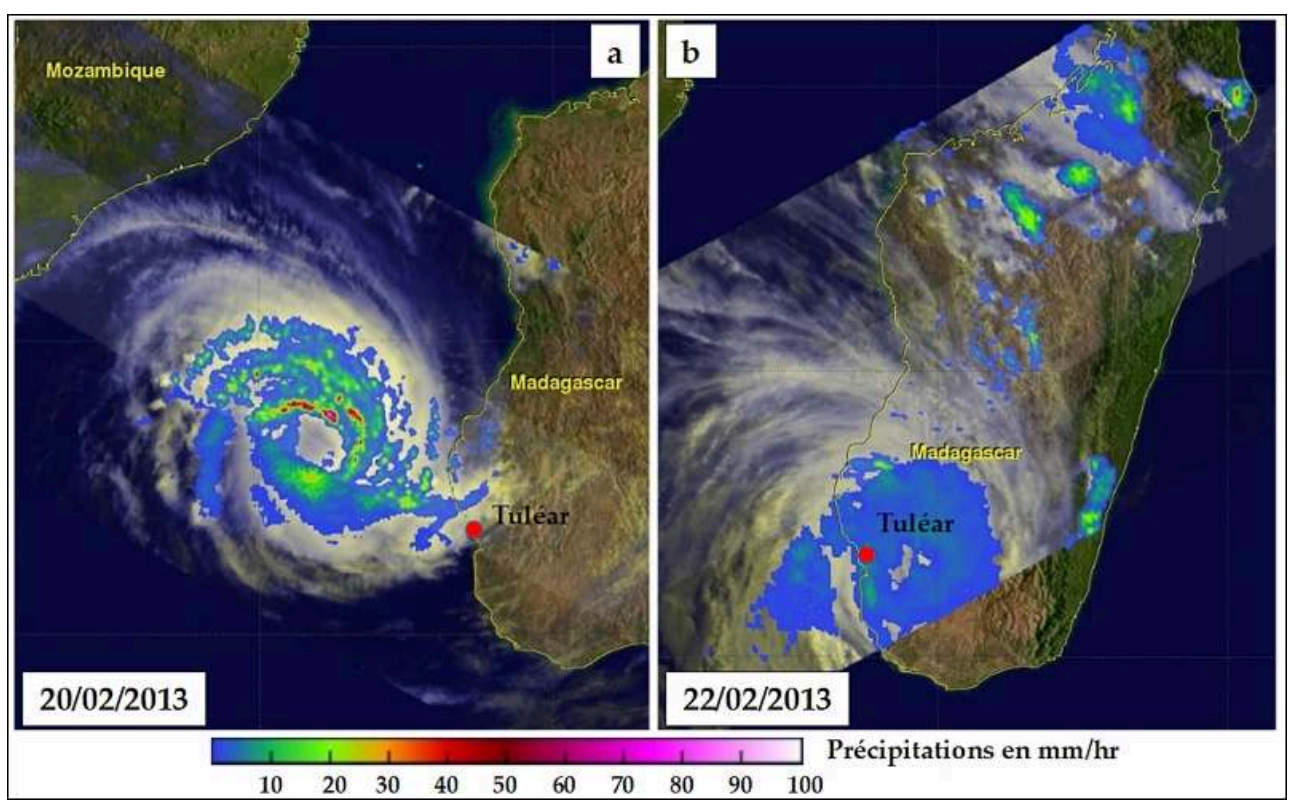

Source : mesure des précipitations (en mm/heure) via le satellite TRMM (Tropical Rainfall Measuring Mission) de la NASA (modifié d'après la NASA).

\section{Apport de l'imagerie haute-résolution pour le suivi de l'impact d'Haruna}

Cette étude a bénéficié des ressources de la station SEAS-OI (Surveillance de l'Environnement Assistée par Satellite pour l'Océan Indien). Située à Saint-Pierre de La Réunion, elle a été opérationnelle de juillet 2012 à décembre 2016. Parmi les objectifs de la station SEAS-OI, on peut citer l'acquisition d'images satellites en tâche de fond sur une zone de $2500 \mathrm{~km}$ de rayon autour de La Réunion (incluant Madagascar, illustration 7), et la mise à disposition rapide et gratuite de ces données aux partenaires institutionnels et scientifiques de la sous-région. Grâce à la réception directe d'imagerie SPOT 5 et RADARSAT-2, il a été possible de réaliser un suivi en quasi temps réel de 
l'évolution des inondations causées par Haruna entre le 20 et le 26 février. Ce suivi a été mené suite à une sollicitation du BNGRC.

Illustration 7 - Cercle de réception directe des données SPOT-5 et RADARSAT-2 de la station SEASOl

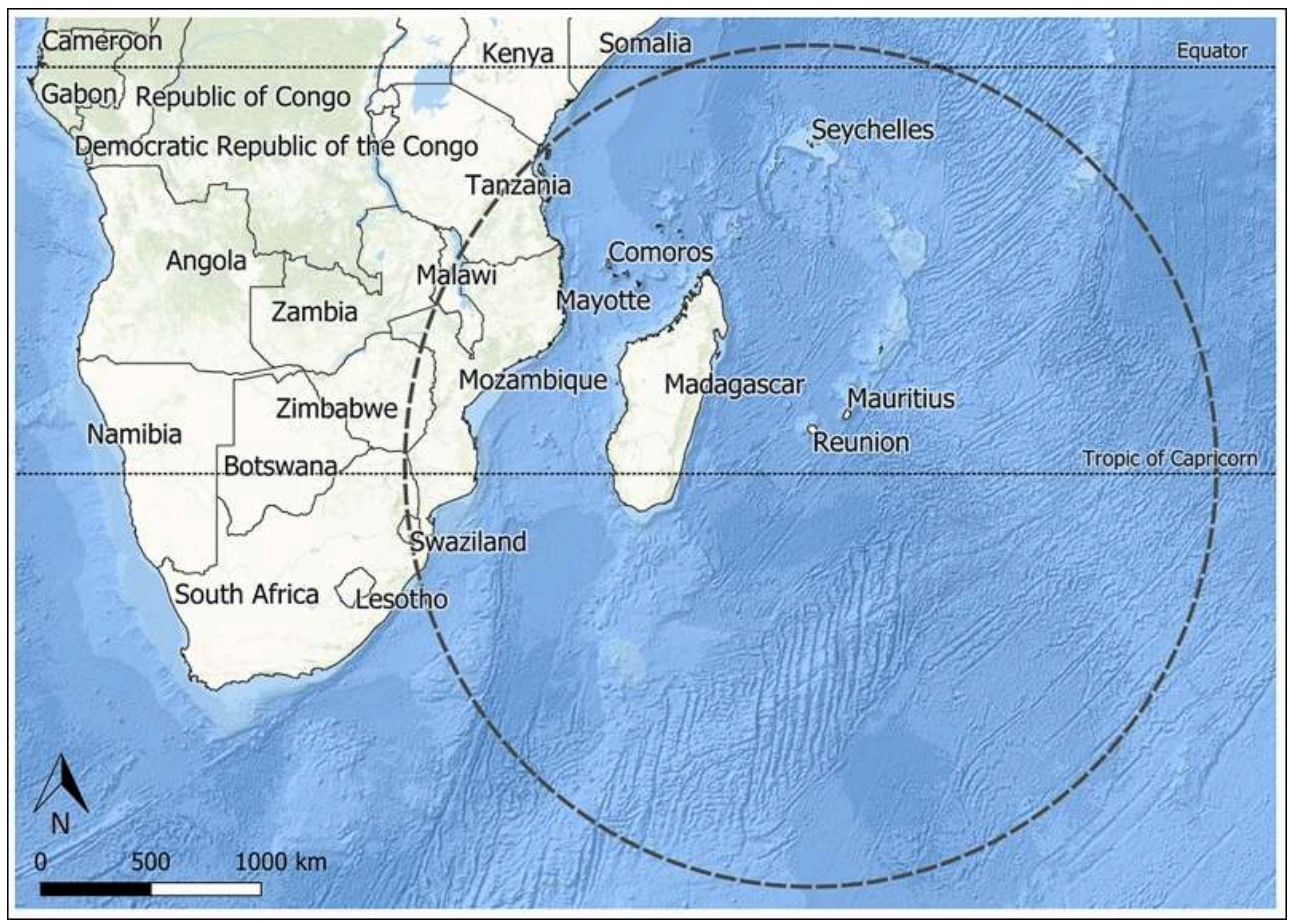

Source : Fonds ocean Basemap, (C) Esri. Auteur : C. Révillion.

L'intérêt de la station SEAS-OI au moment de l'étude réside dans la maitrise de la programmation, du stockage et dans l'acquisition combinée de données d'observation de la Terre issues de capteurs optique (SPOT 5) et SAR (Synthetic Aperture Radar, ou RSO, radar à synthèse d'ouverture) (RADARSAT-2). En effet, au pic d'intensité du cyclone le 22 février, l'ennuagement lié au phénomène rend impossible l'observation des territoires affectés par le cyclone par des satellites optiques. RADARSAT-2, capteur actif en bande- $C$ peut quant à lui acquérir des données peu perturbées par l'ennuagement. L'imagerie RADAR, obtenue via une programmation d'urgence initiée par SEAS-OI, permet donc d'obtenir de l'information sur la situation au sol, et de travailler sur l'impact du cyclone, notamment en termes d'extension des zones d'inondation. La méthodologie utilisée pour extraire les pixels inondés à partir d'images RADARSAT-2 à $8 \mathrm{~m}$ de résolution spatiale (mode Fine, polarisation VV+VH) est détaillée dans l'illustration 8 . L'intérêt d'utiliser des images en double polarisation réside dans la possibilité d'accéder à trois informations spectrales (3 canaux) : une information fournie par VV, une information fournie par $\mathrm{VH}$ et une information fournie par le ratio $\mathrm{VV} / \mathrm{VH}$. Ici, la polarisation $\mathrm{VH}$ a été privilégiée dans la mesure où les structures horizontales comme l'eau présentent une forte rétrodiffusion dans la polarisation $\mathrm{VH}$, facilitant l'identification des zones inondées. 
Illustration 8 - Méthodologie de traitement des images RADARSAT-2 pour l'extraction des pixels en eau

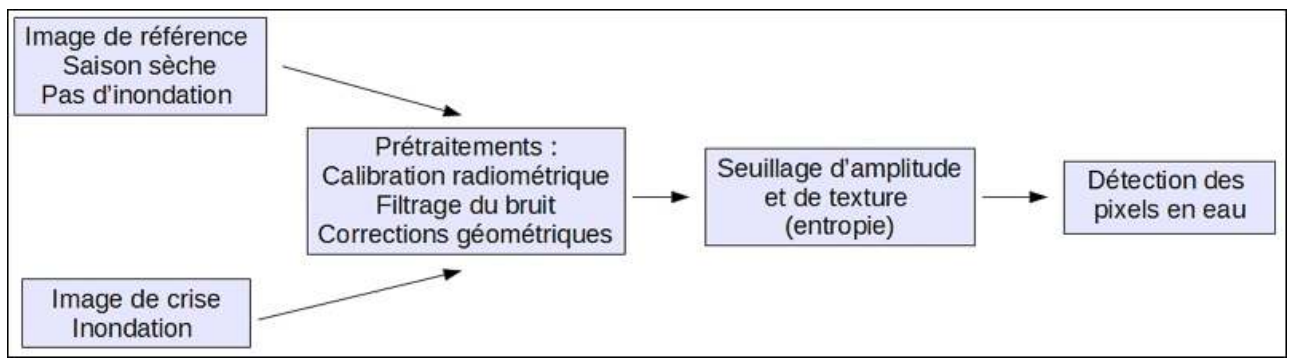

Réalisée à l'aide de la Sentinel Tool Box, SNAP Sentinel Application Platform.

Une image de référence, acquise en saison sèche, correspondant à la situation en absence d'inondation, est utilisée pour la détection et la cartographie des zones en eau permanentes, notamment les fleuves. Dans cette étude, une image datant du 4 juillet 2012 a été utilisée. Cette image permet d'extraire le niveau minimal d'eau observé dans le fleuve. Pour caractériser la situation de crise et l'extension des inondations, une image du 22 février 2013 (pic d'intensité du cyclone, l'œil étant alors situé à $50 \mathrm{~km}$ de Toliara) a été utilisée. A partir de cette image, les prétraitements classiques ont été appliqués (calibration radiométrique, filtrage du bruit puis corrections géométriques) et un seuillage de l'amplitude du signal SAR, combiné à des informations texturales d'entropie (Kiage et al., 2005 ; McMillan et al., 2006 ; Mason et al., 2010) a permis d'identifier les pixels en eau correspondant (i) au fleuve Fiherenana en crue (en bleu illustration 9) et (ii) les autres surfaces en eau (ou inondées) dans la plaine littorale (en rouge Illustration 9) la zone urbaine de Toliara (en jaune illustration 9). L'entropie est un paramètre physique qui caractérise le degré aléatoire de l'orientation spatiale de l'onde rétrodiffusée. Une forte entropie correspond à des structures ayant une géométrie en trois dimensions (des bâtiments en zones urbaines par exemple) qui vont rétrodiffuser un signal dans toutes les directions de l'espace alors qu'une surface lisse comme l'eau ne va rétrodiffuser le signal que dans une seule direction avec une entropie faible. 
Illustration 9 - Cartographie de la zone inondée au pic d'intensité du cyclone Haruna sur la zone de Toliara, Madagascar

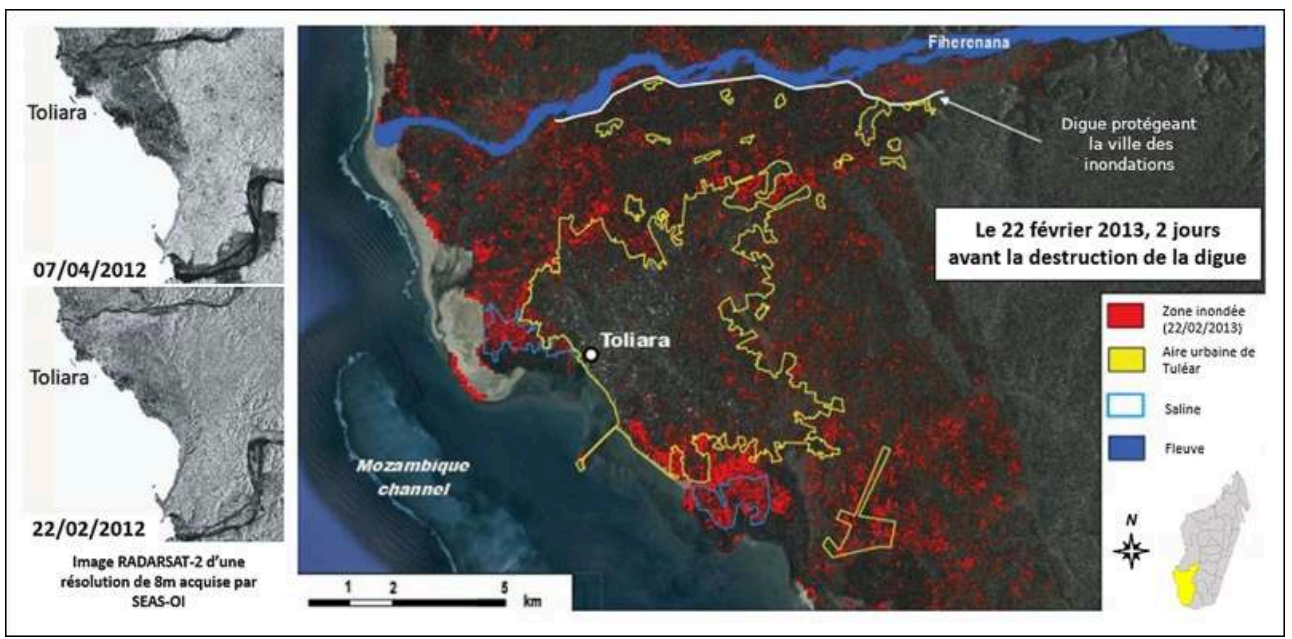

Zone inondée le 22/02/2013 en rouge, cartographiée par imagerie RADARSAT-2.

Source: RADARSAT-2 Data and Products, (C) MacDONALD, DETTWILER AND ASSOCIATES LTD. $(2012,2013)$ - All rights reserved, RADARSAT is an official trademark from the Canadian Space Agency. Auteur : T. Catry.

Il est important de noter qu'à ce stade de la crise, la digue protégeant Toliara des crues du Fiherenana n'a pas encore cédé sous l'augmentation rapide du débit du fleuve et en raison de sa fragilisation localement d'origine anthropique. Les inondations dans la zone de Toliara se limitent à la périphérie de la zone urbaine (en jaune illustration 9) et à la zone côtière. Elles sont la conséquence des fortes pluies et de la houle cyclonique. A partir du 23 février, le niveau d'alerte baisse (illustration 5) mais les pluies se poursuivent, augmentant le débit et le niveau du fleuve. Le 24 février, la digue (en blanc illustration 9) cède et le fleuve en crue se déverse dans les zones basses en direction de l'agglomération de Toliara.

Dans un second temps, grâce à l'utilisation de plusieurs images SPOT 5 multispectrale à 10 mètres de résolution, il a été possible d'évaluer (i) l'impact de la rupture de la digue par le suivi de l'inondation qui en a résulté ainsi que (ii) le suivi de la décrue. La méthodologie employée est détaillée dans l'illustration 10.

Illustration 10 - Méthodologie de traitement des images SPOT 5 pour l'extraction des pixels en eau

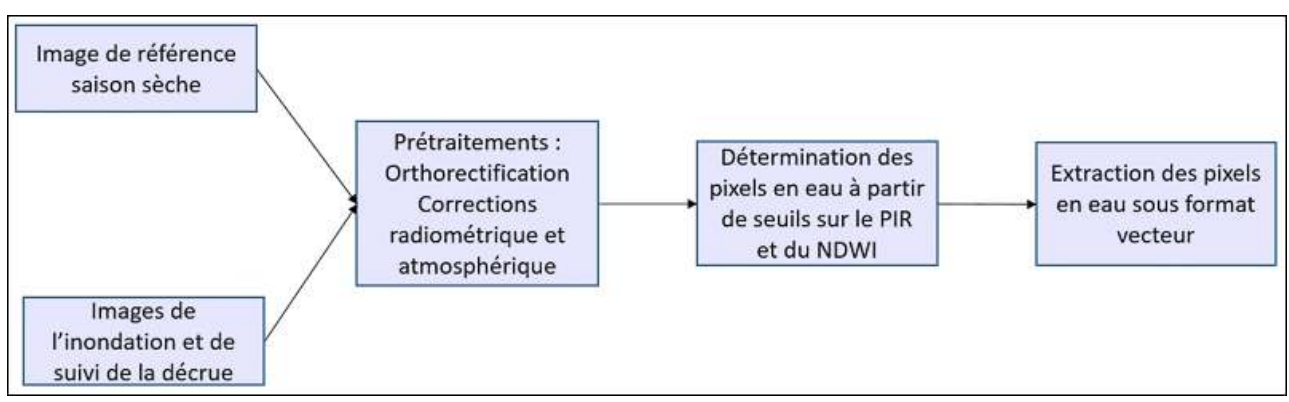

17 Le jeu d'images SPOT 5 qui a été mobilisé pour ce suivi a été téléchargé dans la base de données de la station SEAS-OI, qui disposait d'images de qualité aux périodes et sur les zones concernées. Il s'agit d'une image de référence en saison sèche (acquise le $17 / 08 / 2012)$, d'une image prise quelques jours après le passage du cyclone (26/02/2012) 
et de trois images complémentaires pour suivre la décrue (04/03/2013, 18/03/2013 et 24/04/2013). Ce suivi de la décrue a été réalisé à l'initiative de l'équipe IRD en pied d'antenne à SEAS-OI pour montrer l'intérêt de la répétitivité pour le suivi temporel d'une catastrophe naturelle.

Le jeu d'images a été prétraité avec le logiciel ENVI pour rendre les données superposables (corrections géométriques) et comparables (corrections radiométriques) entre elles. Le NDWI (Normalized Difference Water Index) est un indice de télédétection classiquement utilisé pour mettre en évidence et délimiter les masses d'eau libres. Il est calculé comme la différence normalisée entre les bandes spectrales verte et proche infrarouge (PIR), avec une gamme de valeurs comprises entre -1 et +1 , négatives pour la végétation terrestre et les sols nus, positives dans l'eau (McFeeters, 1996). Pour chaque image du jeu de données, un seuillage du NDWI et de la bande proche infrarouge ont permis d'extraire une classe « zones en eau » (Wang, 2004).

Le climat de Toliara étant sub-aride, la donnée en saison sèche donne une bonne idée de la faible extension des zones en eau durant une bonne partie de l'année (Battistini, 1996). Utilisée comme donnée de référence, elle permet par comparaison avec l'image $\mathrm{du}$ 26/02/2013 de visualiser l'étendue de l'inondation suite aux très fortes précipitations et à la rupture de la digue (illustration 11).

Illustration 11 - Cartographie par imagerie SPOT 5 des inondations dans la zone de Toliara le $26 / 02 / 2013$

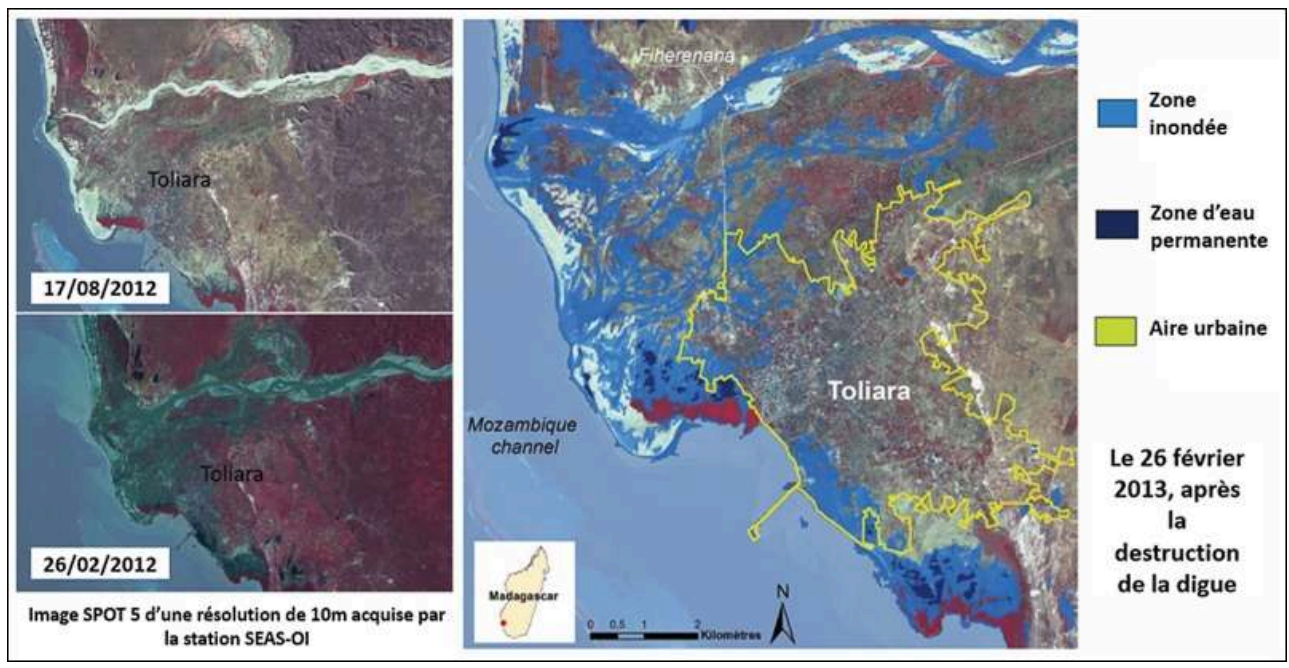

Inondations en bleu clair, après la rupture de la digue protégeant la zone urbaine, en jaune, des crues du Fiherenana.

Source : @ CNES (2012, 2013), Astrium Services Distribution/Spot Image S.A., France, all rights reserved. Auteur: C. Révillion.

En répliquant la méthode décrite à l'illustration 10, un suivi de la décrue a été réalisé au cours des mois de mars et d'avril en utilisant trois images SPOT 5 datées respectivement du 4 mars, 18 mars et 24 avril (illustration 12). Cette approche montre l'intérêt de mettre en place un suivi post-crise afin d'évaluer la résilience d'une zone impactée par un évènement cyclonique majeur. Ce type de suivi n'est pas mis en place dans le cadre de l'activation des chartes risques internationales dans la mesure où il s'agit d'actions d'urgence. 
Illustration 12a - Suivi de la décrue dans la zone de Toliara par imagerie SPOT 5 datant du 04/03/2013 (a), 18/03/2013 (b) et 24/04/2013 (c)

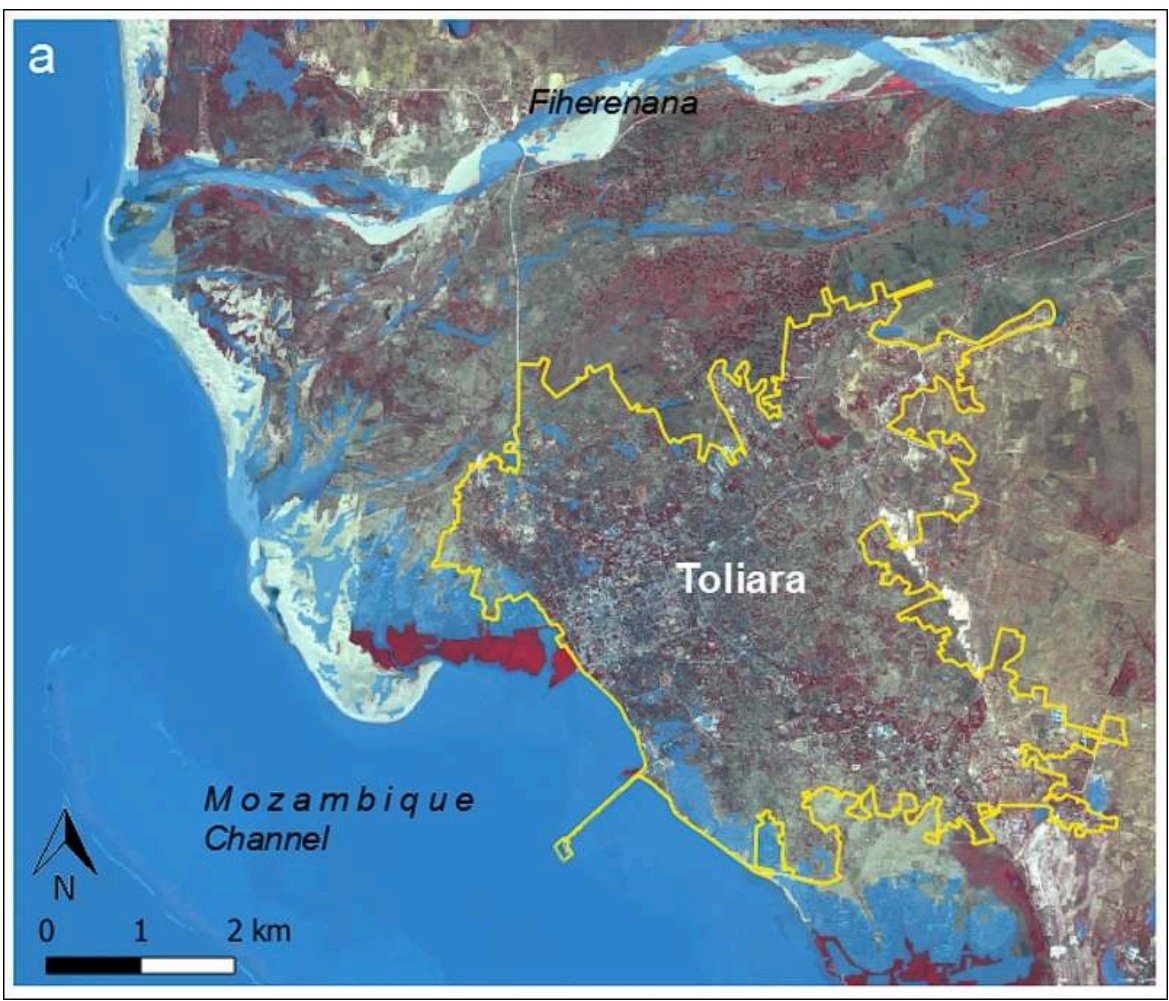

Source : ( C CNES (2013), Astrium Services Distribution/Spot Image S.A., France, all rights reserved. Auteur: C. Révillion. 
Illustration 12b - Suivi de la décrue dans la zone de Toliara par imagerie SPOT 5 datant du $18 / 03 / 2013(b)$

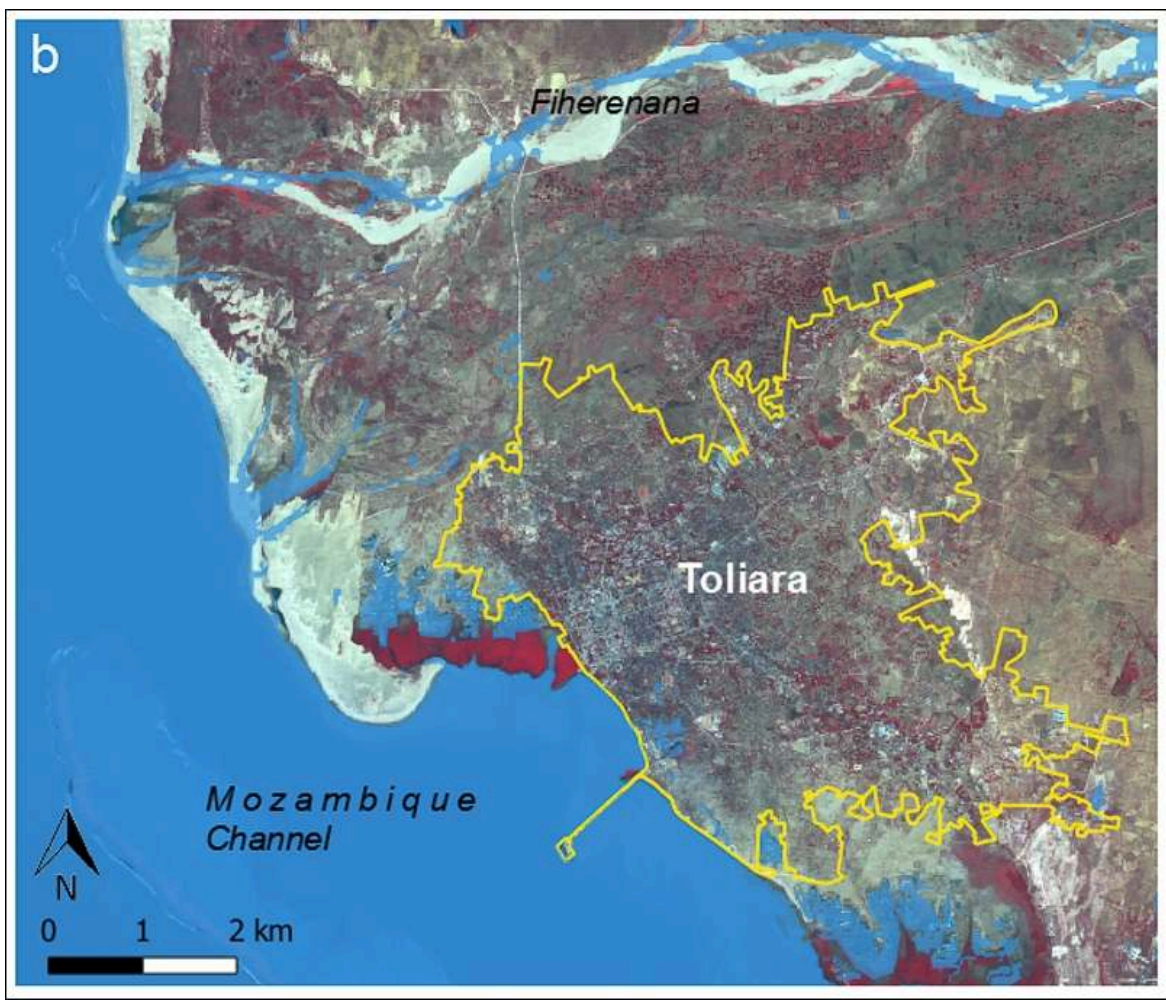

Source : ( ) CNES (2013), Astrium Services Distribution/Spot Image S.A., France, all rights reserved Auteur: C. Révillion. 
Illustration 12c - Suivi de la décrue dans la zone de Toliara par imagerie SPOT 5 datant du $24 / 04 / 2013$

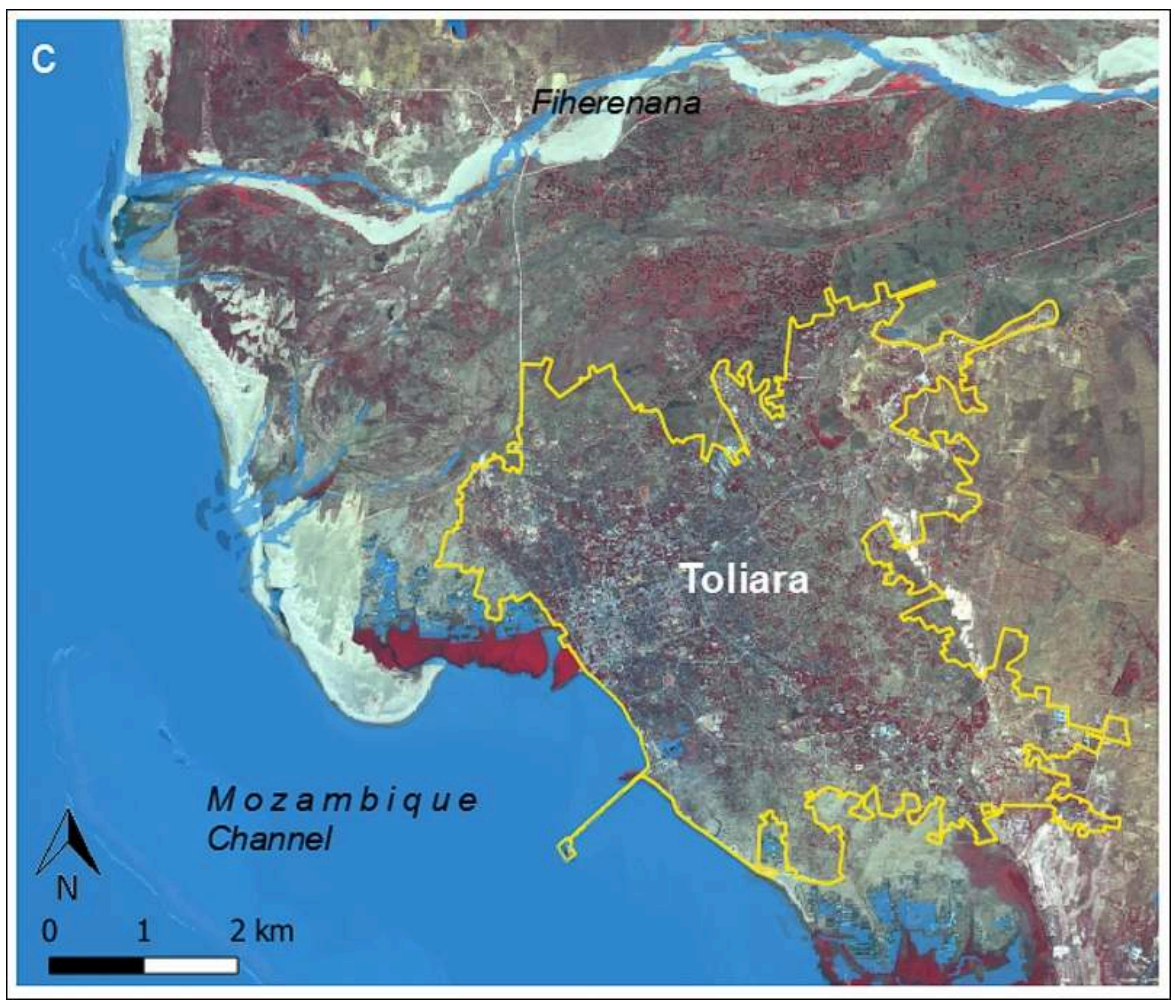

Source : ( C CNES (2013), Astrium Services Distribution/Spot Image S.A., France, all rights reserved. Auteur: C. Révillion.

21 On observe une décrue assez lente, notamment dans certaines zones péri-urbaines ou le niveau d'eau avait atteint plusieurs mètres selon la presse locale. La région de Toliara est une région semi-aride, les précipitations y sont donc très faible en moyenne. C'est pourquoi la ville de Toliara n'a pas de réseau d'évacuation des eaux dimensionnés pour de fortes précipitations, ce qui explique en partie la longueur de cette décrue.

\section{Contribution à l'évaluation du bâti impacté en mobilisant des données Pléiades}

22 Une quantification du bâti impacté à l'échelle d'un quartier de Toliara a été réalisée (illustration 12) via l'utilisation d'une image Pléiades à $70 \mathrm{~cm}$ de résolution du 26 février 2013, acquise et mise à disposition par l'équipex GEOSUD (http://ids.equipexgeosud.fr/).

23 Cette image à très-haute résolution permet de qualifier le type d'occupation du sol affecté par les zones en eau (extraites à partir de SPOT 5). Une approche par photointerprétation est ici privilégiée. L'illustration 13 montre qu'un grand nombre de bâtiments ont été impactés par les inondations. Cette image permet également de mettre en évidence que les zones impactées sont principalement les zones périurbaines, le centre-ville de Toliara ayant été plutôt épargné par le phénomène. 
Illustration 13 - Étendue des inondations et cartographie des bâtiments impactés ou non impactés par les inondations le 26 février 2013

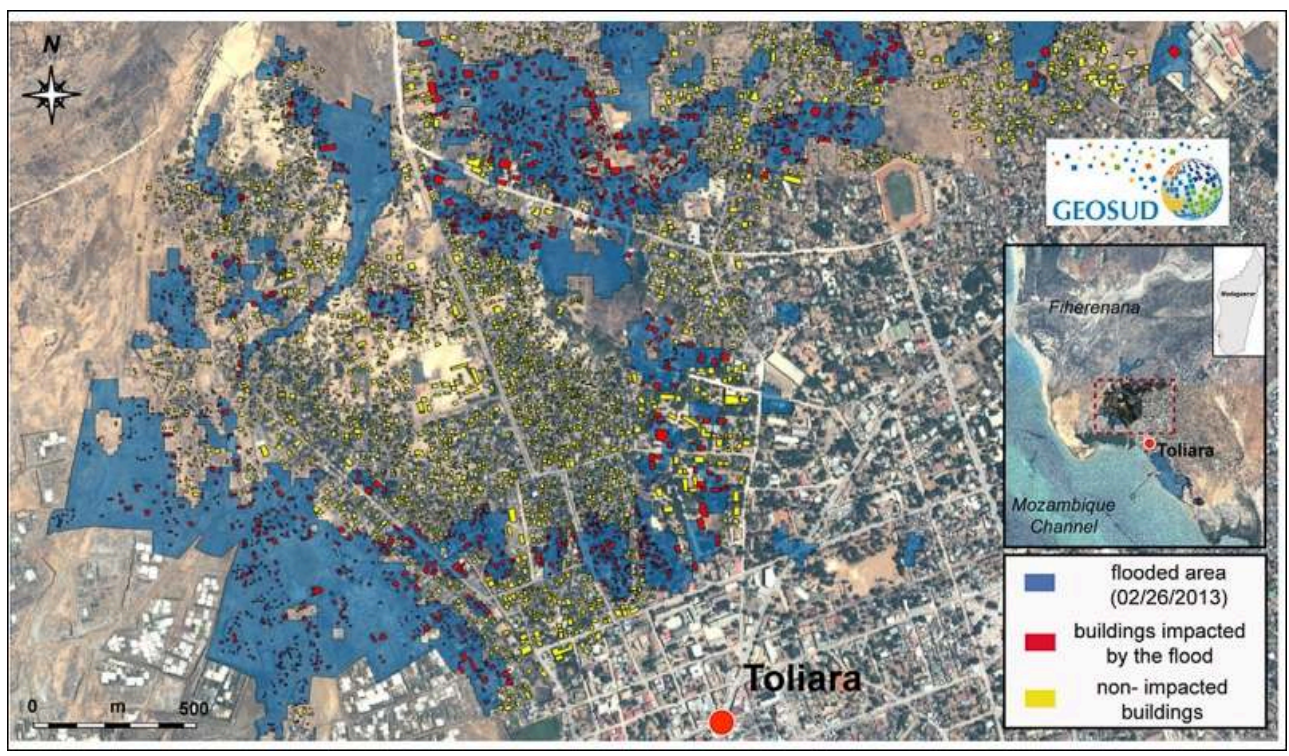

Inondations en bleu (extrait du traitement réalisé sur l'image SPOT 5) ; bâtiments impactés en rouge et non impactés en jaune.

Source : contient des informations ( C CNES 2013, Distribution Airbus DS, tous droits réservés. Usage commercial interdit. Auteur : C. Révillion.

\section{Bilan du cyclone Haruna}

La combinaison de capteurs optiques, RADAR pluie et RADAR imageur SAR à différentes résolutions a permis de réaliser un suivi complet du développement du cyclone Haruna puis de ses conséquences en terme d'inondation et d'impact sur les infrastructures (Chaouch et al., 2012), en lien étroit avec les autorités malgaches, via le BNGRC qui a sollicité l'appui de la station SEAS-OI pour la gestion de crise. Durant ce cyclone, si les autorités malgaches signalaient 41000 personnes sinistrées (26 décès, 16 disparus, 127 blessés, 13800 sans-abris), le suivi par imagerie satellite montre que 15000 habitations auraient été directement impactées par la montée des eaux. Sur cet événement, la charte internationale Espace et catastrophes majeures a été déclenchée par la protection civile française à la demande du BNGRC. Dans ce cadre, le SERTIT a réalisé une carte des zones inondées très rapidement après l'événement. La cartographie des zones en eau réalisée par le SERTIT et celle élaborée dans cette étude se fondent sur la même image SPOT 5 du 26 février 2013.

Même si les objectifs ne sont pas les mêmes, le résultat des deux traitements est assez proches. Le travail du SERTIT étant de proposer un produit opérationnel dans des délais restreints, nous avons réalisé des analyses complémentaires en suivant la décrue et en estimant le nombre de bâtiment touchés par les inondations. 


\section{Cas d'étude $\mathrm{n}^{\circ} 2$ : le cyclone Enawo de mars 2017, côte nord-est de Madagascar}

\section{Observation du cyclone par imagerie basse résolution}

Les provinces d'Antsiranana et Tamatave sont frappées par le cyclone Enawo en mars 2017. L'illustration 14 montre des images MODIS (capteur de la NASA sur les satellites Aqua et Terra) à $1 \mathrm{~km}$ de résolution spatiale d'Enawo les 6 (gauche), 7 (centre) et 8 (droite) mars 2017, sur lesquelles les sites d'étude, les villes de Sambava et Maroantsetra, sont positionnés.

Illustration 14 - Images satellites MODIS à $1 \mathrm{~km}$ de résolution spatiale du cyclone Haruna les 6 (gauche), 7 (centre) et 8 (droite) Mars 2017

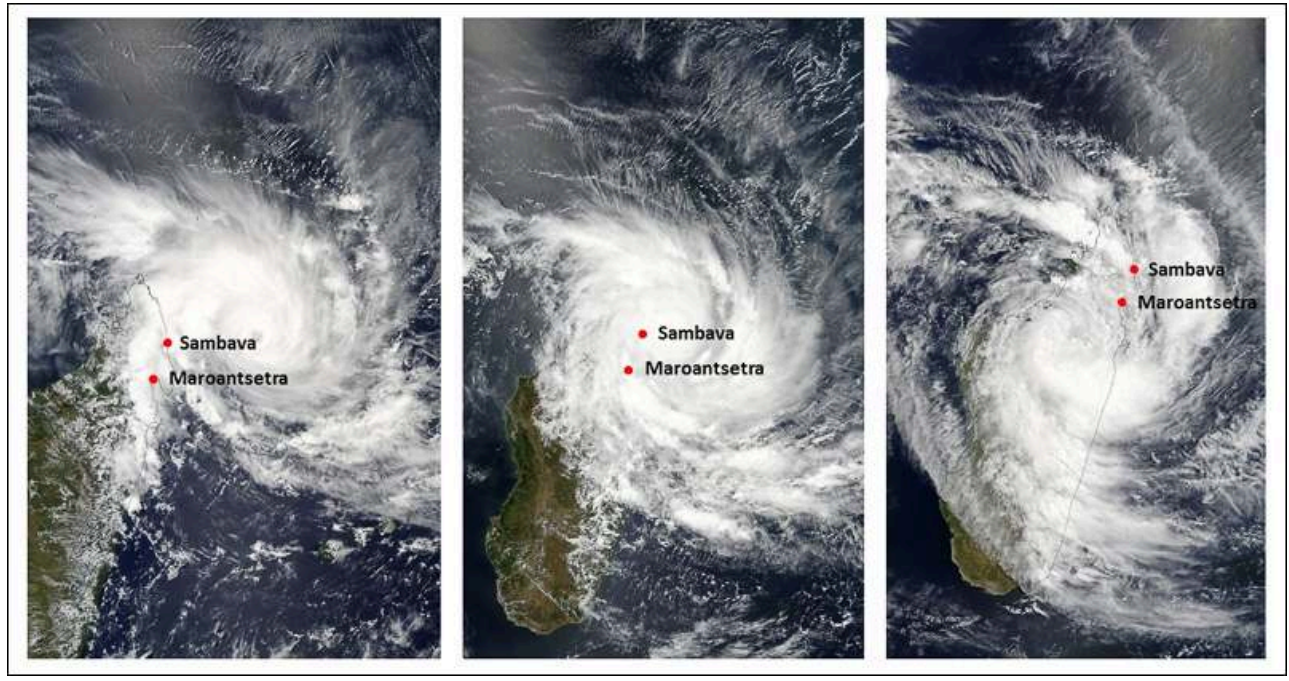

Source: NASA.

\section{Quantification des précipitations associées à Enawo par imagerie satellite}

Grâce au capteur IMERG (micro-ondes RADAR et infra-rouge) de la Global Precipitation Mission (GPM, qui a succédé à TRMM), il a été possible d'avoir une approche quantitative des précipitations totales cumulées au cours du déplacement du cyclone Enawo entre le 3 et le 8 mars 2017. Elles atteignent un maximum de $500 \mathrm{~mm}$ en mer au nord-est de Madagascar et d'environ 200 à $300 \mathrm{~mm}$ au niveau des zones de Sambava et Maroansetra touchées entre les 7 et 8 mars (illustration 15). 


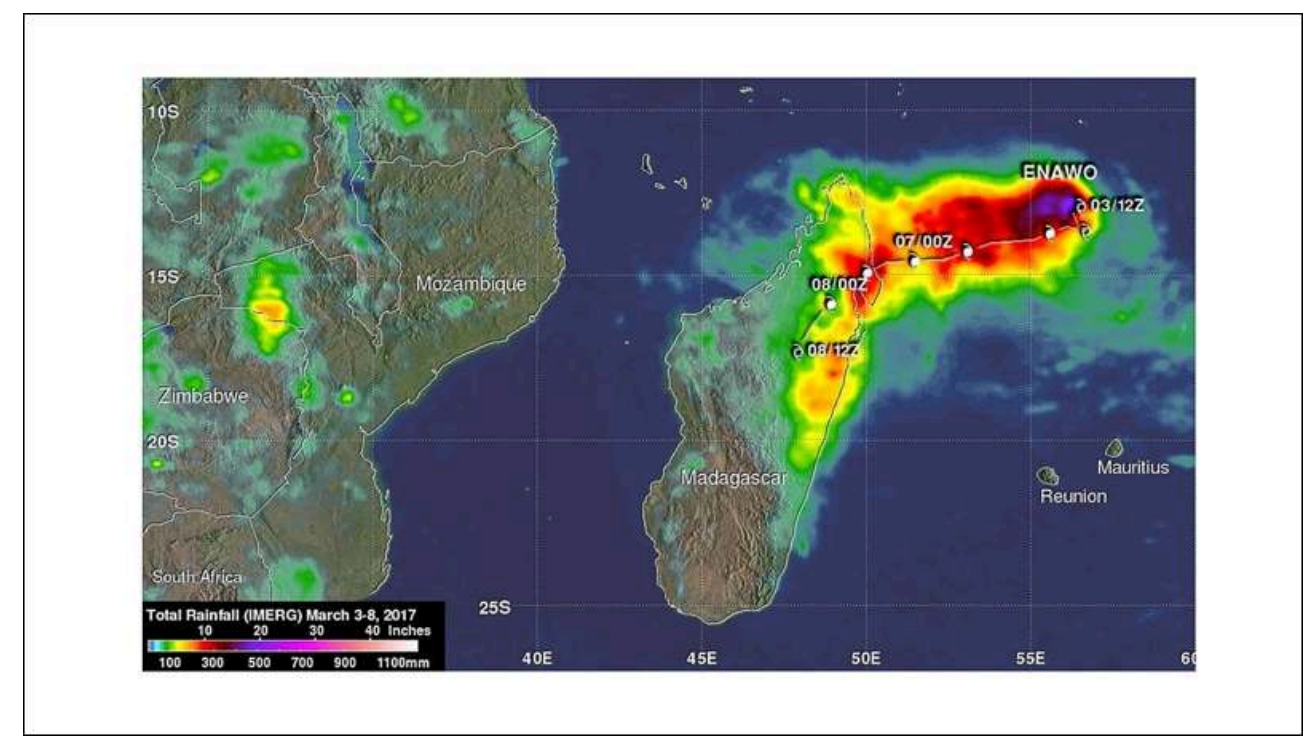

Précipitations totales en mm, via le capteur IMERG de la Global Precipitation Mission (GPM).

Source : NASA.

Le caractère exceptionnel de cet événement a amené les autorités à craindre un impact humanitaire important. C'est pourquoi, pour organiser au mieux l'action des secours et l'aide humanitaire l'EMS (Emergency Management Service) Rapid Mapping de Copernicus a été mobilisé. Dans ce cadre, les cartographies ont été réalisées par la société SIRS (Systèmes d'Information à Référence Spatiale) dans le consortium e-GOES. La charte internationale espace et catastrophes majeures a également été déclenchée par UNITAR-UNOSAT pour UNOCHA, avec réalisation de produits cartographiques par UNOSAT.

\section{Cartographie des inondations sur Maroantsetra par imagerie RADAR Sentinel-1}

Dans le cadre du programme Copernicus (ex-GMES), l'ESA (European Space Agency) met en accès libre depuis 2015 des images satellites optiques Sentinel-2 (S2) et SAR Sentinel-1 ( $\mathrm{S} 1$ ) pour la communauté d'utilisateurs. Ces images ont une résolution spatiale de $10 \mathrm{~m}$ pour $\mathrm{S} 2$ et de $5 \mathrm{~m}$ à $20 \mathrm{~m}$ pour $\mathrm{S} 1$ et une répétitivité temporelle de 5 à 12 jours en fonction de la zone d'étude. Les images S2 sont acquises de façon systématique au nadir, et selon des plans de programmation prévus à l'avance pour S1, non accessibles aux reprogrammations en urgence. Dans le cadre de cette étude, une cartographie des inondations liées à Enawo est réalisée sur la zone de Maroantsetra à partir de trois images Sentinel-1 à $10 \mathrm{~m}$ en polarisation VV + VH. La méthode est la même que celle utilisée précédemment sur les images RADARSAT-2 de Toliara, en utilisant la Sentinel Tool Box de SNAP, logiciel libre fourni par l'ESA (illustration 8). L'image de référence correspondant à la situation en absence d'inondation en saison sèche date du 1/10/2016 (illustration 16a). Une image du 22/02/2017, pré-cyclone mais en saison humide (illustration 16b), est utilisée pour quantifier la présence d'eau liée aux pratiques agricoles, la zone étant une zone rizicole. Enfin, une image en contexte cyclonique dite «de crise» datant du 12 mars 2017 permet de cartographier les 
inondations cycloniques. Sur cette image, les zones en noir (à forte réflexion spéculaire) correspondent aux zones en eau suite aux inondations (illustration 16c). Contrairement au cas précédent (mobilisant des données RADARSAT-2) le mode programmation n'est pas possible avec Sentinel-1, nous sommes donc tributaires des dates d'acquisition des images et des délais (en général 24h) de mise à disposition de la donnée. L'image de crise utilisée date du 12 mars alors que le cyclone est passé sur zone entre le 7 et le 8 mars.

Illustration 16 - Images Sentinel-1A de la zone de Maroantsetra (Madagascar)

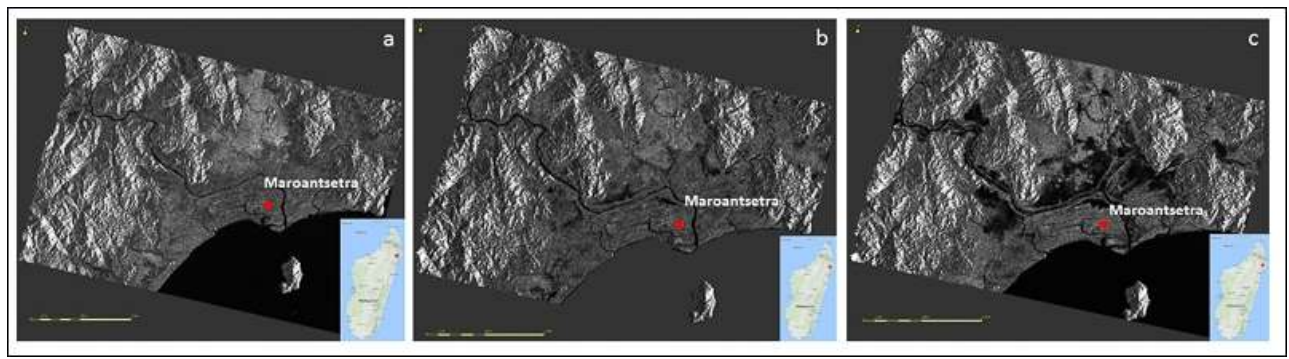

(a) 01/10/2016, pré-cyclone Enowa, saison sèche ; (b) 22/02/2017, pré-cyclone Enowa, saison des pluies ; (c) 12/03/2017, post-cyclone Enowa.

Polarisation $\mathrm{VH}$, résolution $10 \mathrm{~m}$.

(C) ESA

30 Ces trois images ont permis d'obtenir une cartographie fine des zones en eau suite au passage du cyclone Enawo sur Maroantsetra (illustration 16a, b et c). En violet sont figurées les zones en eau permanente (fleuve), en orange les rizières inondées et en rouge les inondations cycloniques. L'illustration 17 montre que le centre-ville de Maroantsetra a été relativement épargné, contrairement à sa périphérie et aux zones agricoles plus au nord qui subissent une forte inondation.

Illustration 17 - Cartographie par imagerie Sentinel-1 des inondations (en rouge) liées au passage du cyclone Enawo sur la zone de Maroantsetra

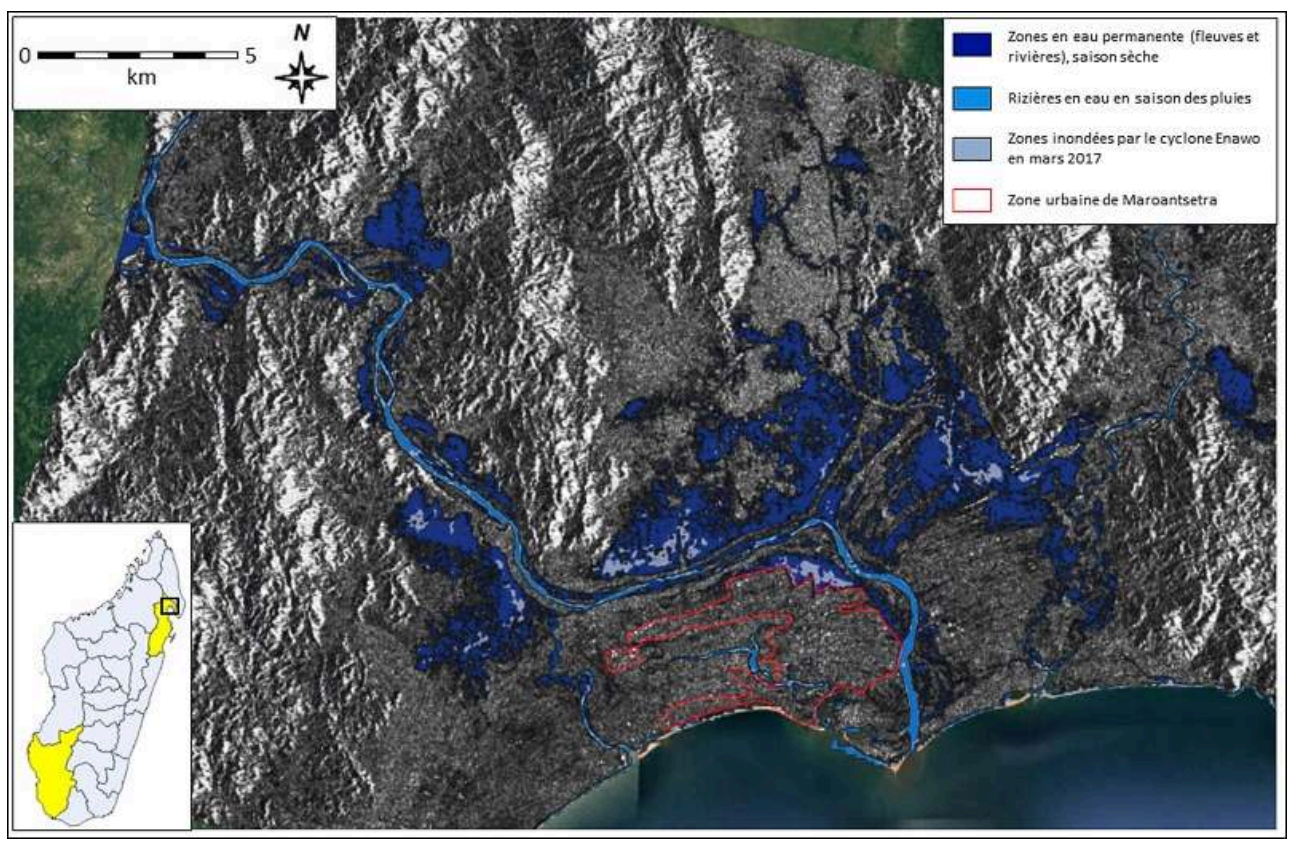

Fond Google Earth. Auteur : T. Catry. 


\section{Cartographie des inondations liées à Enawo sur la zone de Sambava par imagerie optique Sentinel-2}

31 Deux images Sentinel-2 ont été utilisées pour évaluer l'étendue des zones encore en eau cinq jours après le passage du cyclone (illustration 18). Une image de référence acquise un mois avant l'événement donne une information précise sur l'étendue normale des zones en eau en cette saison et l'image du 12 mars 2017 montre bien les zones encore inondées cinq jours après le passage de l'événement cyclonique. Les méthodes de prétraitements sur ce jeu de données sont très proches de celles appliquées sur les images SPOT 5 de Toliara (illustration 10).

Illustration 18 - Les deux images Sentinel-2 utilisées pour la cartographie des inondations suite au passage d'Enawo sur Sambava

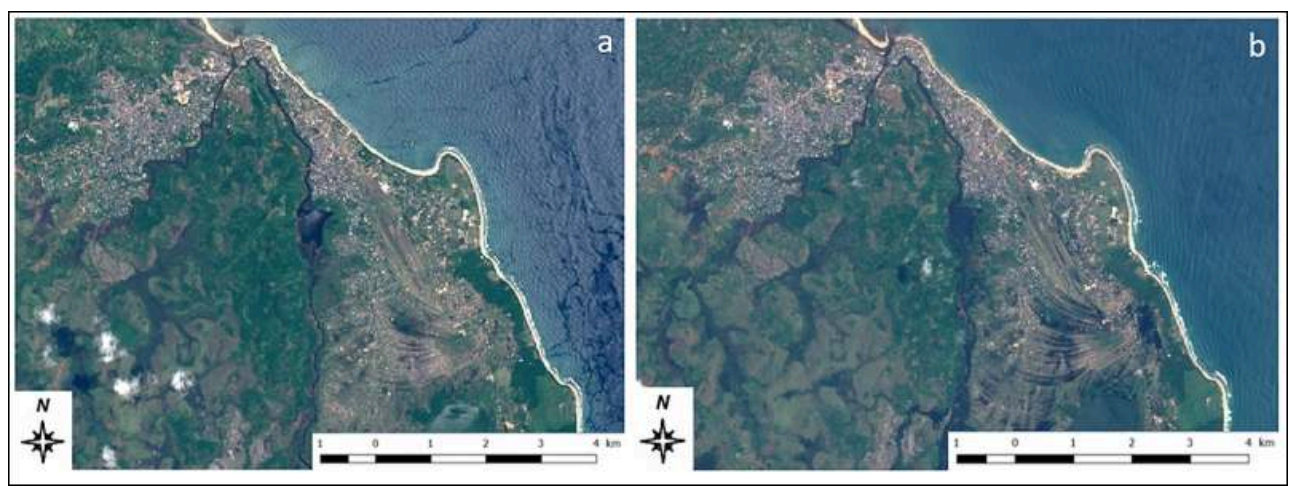

(a) pré-cyclone datant du 10/02/2017 ; (b) post-cyclone du 12/03/2017. Résolution : $10 \mathrm{~m}$ (c) ESA. Auteur : C. Révillion.

Les caractéristiques spectrales des images Sentinel-2 ont permis d'affiner la détection des zones en eau en utilisant les longueurs d'ondes du proche infrarouge (PIR) et du canal moyen infrarouge (MIR) (illustration 19). 
Illustration 19 - Cartographie par imagerie Sentinel-2 des inondations liées au cyclone Enawo sur Sambava cinq jours après le passage du cyclone

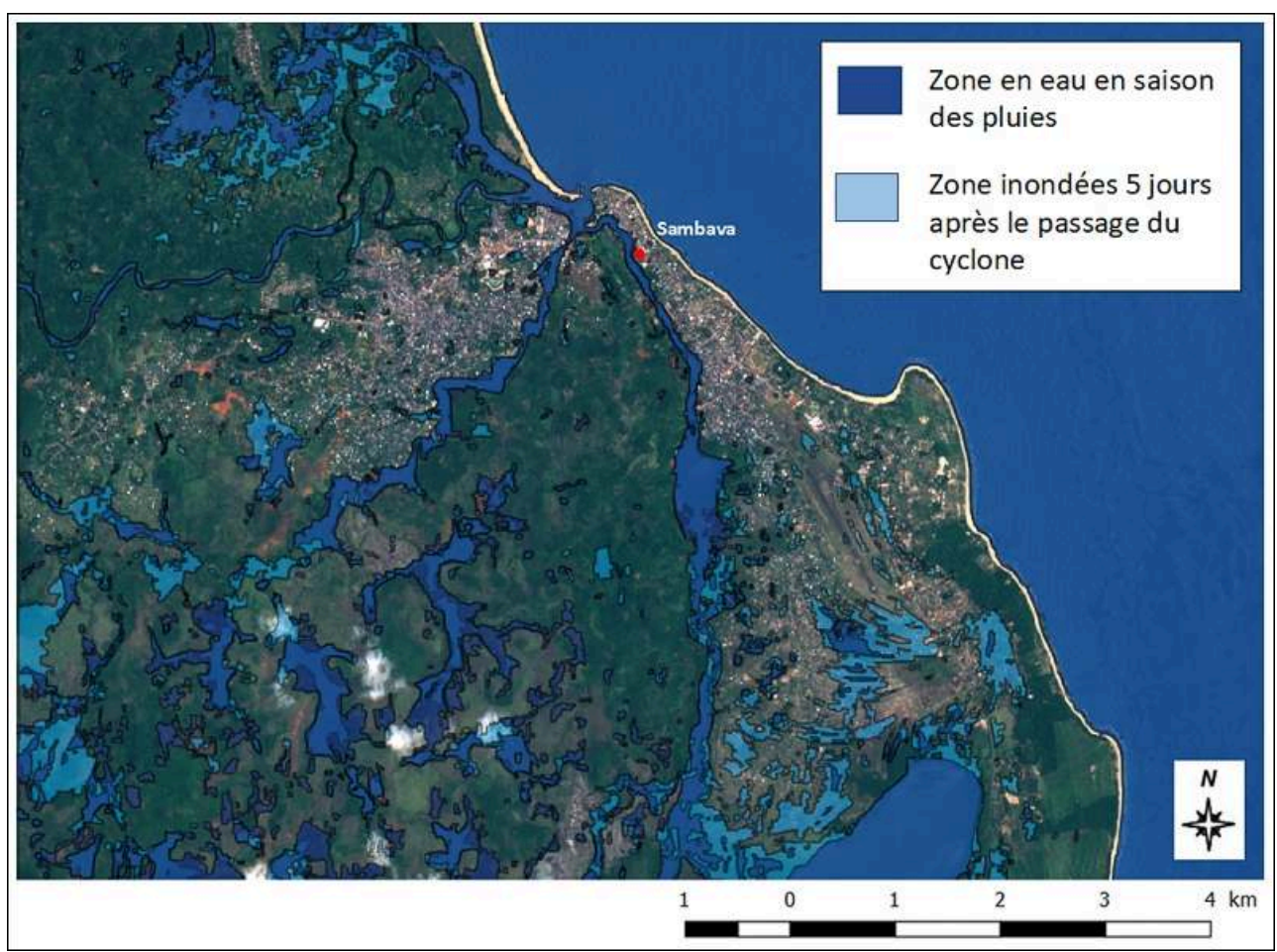

(C) ESA. Auteur : C. Révillion.

La délimitation des zones en eau post événement montre que les surfaces encore inondées se situent surtout au nord et au sud de la ville. Ce sont des espaces de culture périurbaine, notamment des rizières. Le centre-ville est quant à lui peu inondé cinq jours après le passage du cyclone.

34 L'illustration 20 présente une cartographie des inondations liées au cyclone Enawo sur Maroantsetra, réalisée dans le cadre de l'Emergency Management Service (EMS) de Copernicus, à partir d'une combinaison de données Sentinel-2 (pré-cyclone, optique, 10/02/2017), RADARSAT-2 (RADAR, post-cyclone, 7/03/2017, bleu clair) et CosmoSkyMed (RADAR, post-cyclone, 10/03/2017, bleu turquoise). 


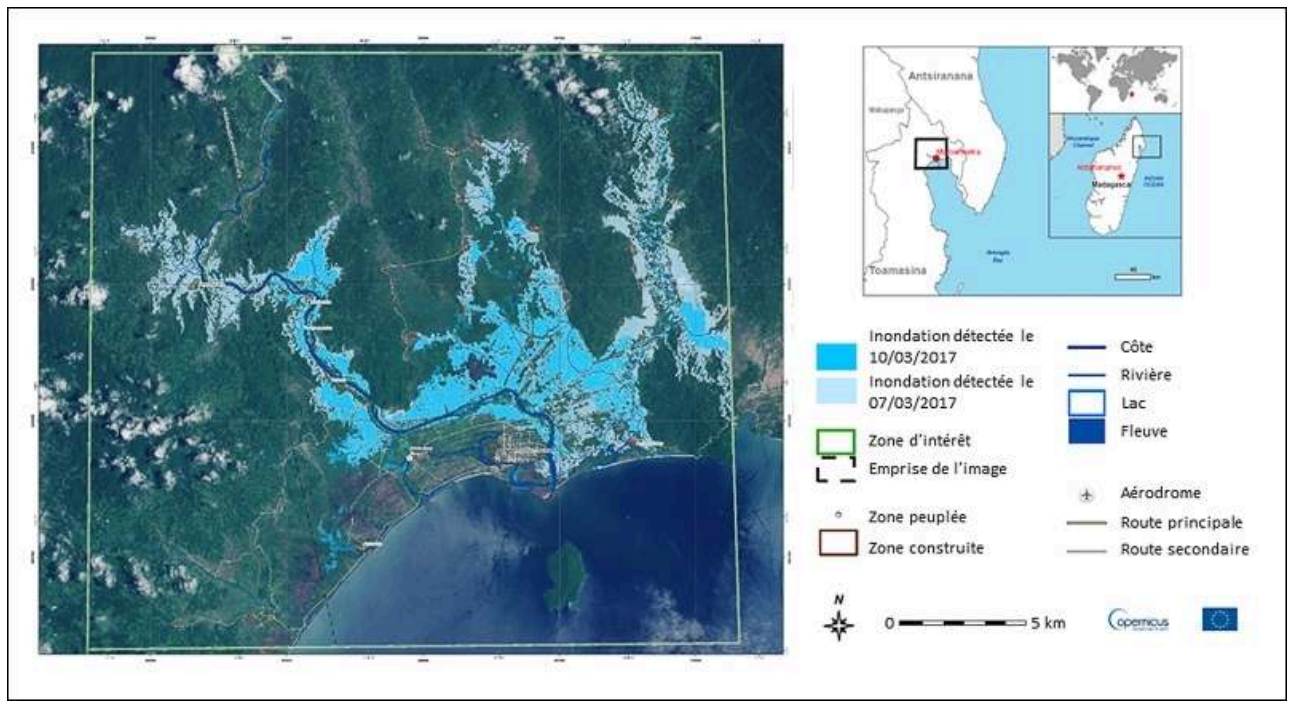

Cartographie via la combinaison de données optique Sentinel-2 et RADAR RADARSAT-2 + CosmoskyMed.

(c) European Union.

Bien que les dates d'acquisition des images soient différentes, les résultats obtenus avec Sentinel-1 datant du 12 mars (illustration 17) sont en accord avec les produits fournis par Copernicus (illustration 20).

\section{Apport d'OpenStreetMap (OSM) et de la très haute résolution spatiale pour la détection des infrastructures impactées}

OSM est un projet collaboratif qui a pour but de constituer une base de données géographiques sous licence libre (ODbL). Toute donnée ajoutée à la base est librement réutilisable. Depuis le début des années 2010 la communauté des contributeurs d'OSM est régulièrement sollicitée pour des actions de cartographie post catastrophes au travers de l'interface HOT (Humanitarian OpenStreetMap Team) dans les pays ne disposant pas de cartographie de référence à grande échelle. Rendu populaire suite à la mobilisation de la communauté lors du tremblement de terre d'Haïti en janvier 2010, il est désormais habituel pour les ONG et les structures responsables de l'aide humanitaire de s'appuyer sur la communauté des contributeurs OSM. En outre, lors de ces actions les providers publics et privés de données satellites mettent à disposition des images THRS pour cartographier au mieux les bâtiments, les réseaux etc. On parle alors de tâche HOT ${ }^{4}$. Suite au passage d'Enawo, une tâche HOT a été déclenchée pour la ville de Sambava et une autre pour le site de Maroantsetra, ces deux projets ont bénéficié d'images GeoEye1 fournies par DigitalGlobe. Cette action a permis une cartographie précise des bâtiments de la zone en quelques jours. En plus d'avoir contribué au déploiement humanitaire sur site, les données bâti de Sambava peuvent être mobilisées pour d'autres études car accessibles sur OSM. L'intersection de la couche «bâti » OSM et de la zone inondée sur Sambava nous a permis d'estimer à 1200 les bâtiments encore touchés par les inondations cinq jours après le passage du cyclone (extrait de l'analyse illustration 21). Ce qui représente environ $10 \%$ des bâtiments de Sambava. Sur Maroantsetra, le constat d'un centre-ville relativement épargné par les inondations est 
confirmé par le calcul du nombre de bâtiment impacté. Grâce à la BDD OSM nous avons calculé que seuls 10 bâtiments avaient encore les pieds dans l'eau quatre jours après le passage du cyclone (environ 1/1000).

Illustration 21 - Localisation des bâtiments issue de la base de données OSM sur le sud de la ville de Sambava

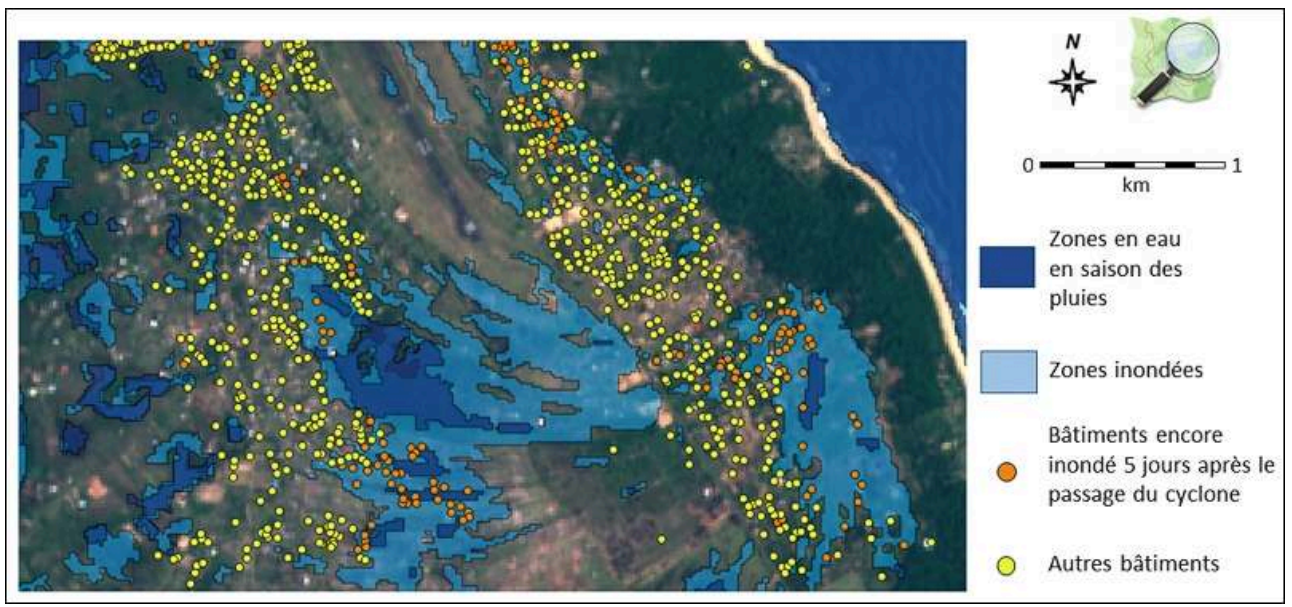

Localisation utilisée pour la cartographie des bâtiments impactés suite au passage d'Enawo.

Auteur: C. Révillion.

37 L'illustration 22 présente quant à elle une cartographie de l'impact des inondations sur les routes et les bâtiments, réalisée à partir d'une image GeoEye DigitalGlobe du 11/03/2017 dans le cadre de l'Emergency Management Service (EMS) de Copernicus, avec deux niveaux d'impact sur les routes et le bâti : faiblement endommagés en jaune et fortement endommagés en orange. Cette figure illustre l'apport de la très haute résolution pour l'estimation des dommages structurels sur des objets dont les dimensions sont trop faibles pour être cartographiables à partir d'images satellites de plus faibles résolutions (Sentinel-2, SPOT 5). 
Illustration 22 - Cartographie des dommages structurels aux routes et bâtiments sur la zone de Maroantsetra

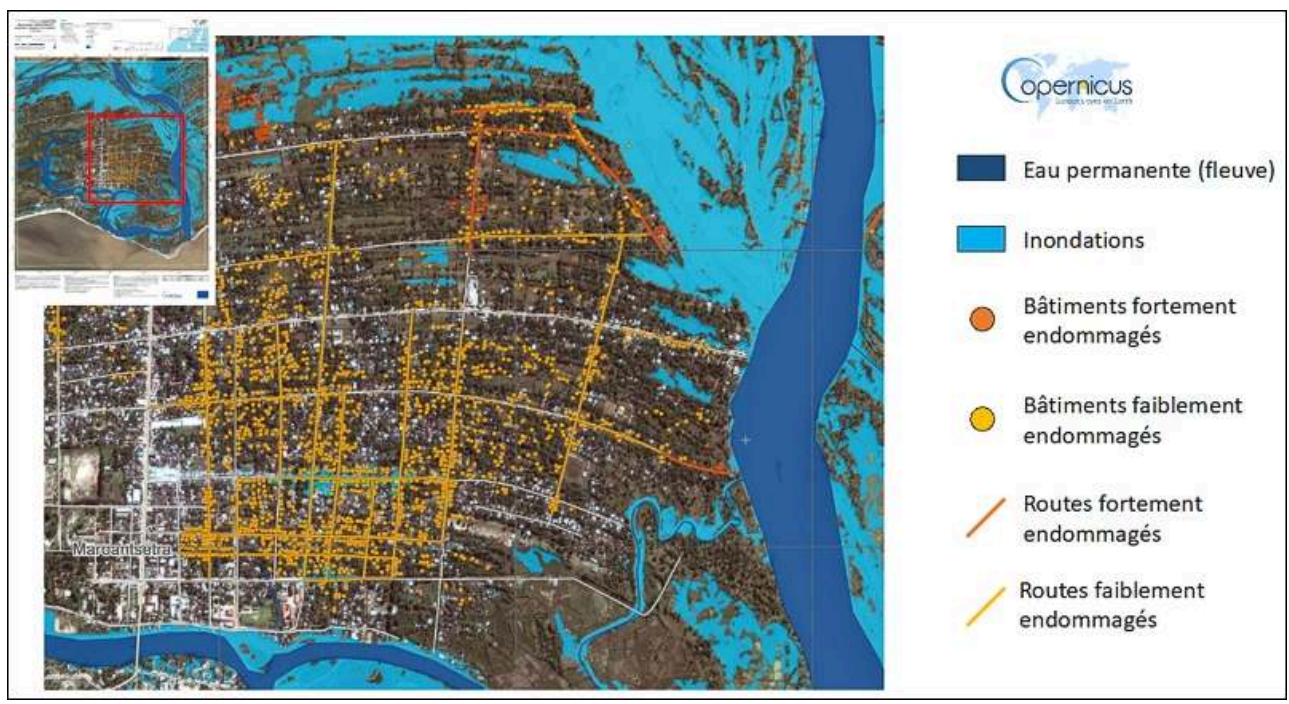

Cartographie réalisée à partir d'une image très haute résolution GeoEye (DigitalGlobe) du 11/03/2017. (c) European Union.

\section{Bilan du cyclone Enawo}

38 La mise à disposition libre et gratuite des données Sentinel-1 et Sentinel-2 par Copernicus a permis de quantifier a posteriori l'impact du cyclone Enawo, grâce à la combinaison d'informations spatialisées extraites des capteurs optiques et RADAR. Environ 400000 personnes ont été sinistrées par Enawo (78 morts, 18 disparus, 250 blessés et 250000 personnes déplacées). Associée à une démarche de cartographie participative type OSM outre l'estimation a posteriori, le suivi a contribué à apporter des informations aux organisations en charge de la gestion de la catastrophe.

\section{Conclusion et perspectives}

Cette étude a bénéficié des ressources offertes par la station de réception directe SEASOI (Surveillance de l'Environnement Assistée par Satellite pour l'Océan indien) située à La Réunion, du programme Sentinel-Copernicus de l'ESA et des images Pléiades du CNES/ADS Intelligence pour la mise à disposition des images satellites. Elle met à profit la forte répétitivité temporelle des images satellites fournies par les capteurs Sentinel notamment, et la combinaison des informations extraites des satellites optiques et RADAR. Que ce soit en quasi temps-réel (Haruna) ou a posteriori (Enawo), la complémentarité des capteurs apparait clairement à la vue de ces résultats, bien que les finalités soient différentes (gestion de crise pour Haruna, étude post-castastrophe pour Enawo). L'imagerie satellite a montré son potentiel pour le suivi des phénomènes extrêmes comme les cyclones ainsi que pour la quantification des impacts sur le terrain (inondations, dommages sur les infrastructures), en mettant à profit l'approche multicapteurs et multi-résolutions. Les résultats obtenus dans cette étude sont complémentaires des travaux menés par le SERTIT (Service Régional de Traitement de l'Information et de Télédétection) dans le cadre de la charte " rapid response » des Nations Unies ou par Copernicus en cartographie rapide via le «Early Management 
Service " (illustrations 21 et 22). Ces études constituent une exploration de l'exploitation des données d'observation de la Terre à différentes résolutions spatiales, temporelles et avec divers modes (optique, radar), pour l'évaluation de l'impact des cyclones dans le sud-ouest de l'océan Indien. L'observation de la Terre est également un atout majeur pour réaliser le suivi du retour à la normale et la dynamique de décrue sur une vaste zone.

Elles ont d'ores et déjà contribué à la mise à jour de l'atlas des risques naturels de Madagascar (Rakotondrasoa et al., 2016). En ce qui concerne les aspects organisationnels de la gestion de crise, il est nécessaire d'anticiper les mécanismes de recours à la Charte et /ou Copernicus Emergency, de programmations d'urgence (via les stations SEAS ou autre...), pour que l'observation de la Terre soit efficace en support aux gestionnaires de crise. En ce sens, les données S1 et S2 assurent une garantie d'accès aux images, mais l'accès ç des données à très haute résolution spatiale via l'activation des chartes internationales reste complètement indispensable pour les cartographies urbaines.

Une approche spatialisée des impacts économiques est mise en place dans la phase suivante avec le programme Interreg Renovrisk-Impact (2018-2021) réunissant une équipe multidisciplinaire de géomaticiens-télédétecteurs, géographes et économistes de l'environnement. Les méthodes décrites dans cet article sont mobilisées et améliorées pour mesurer l'impact matériel et économique des cyclones et étudier la résilience des territoires touchés. Les travaux se basent notamment sur le développement d'une chaîne de traitement semi-automatisée des images Sentinel-1 et 2 (téléchargement des images, mise en cohérence, prétraitements, calculs d'indices et détection de changements par des analyses diachroniques).

\section{BIBLIOGRAPHIE}

Battistini R., 1996. Paléogéographie et variété des milieux naturels à Madagascar et dans les îles voisines : quelques données de base pour l'étude biogéographique de la "région malgache". In Lourenço W.R. ed., Biogéographie de Madagascar. Paris, ORSTOM, p. 1-17.

Brown M. L., 2009. Madagascar's Cyclone Vulnerability 11 and the Global Vanilla Economy. In Jones E. C., Murphy A. D. (ed.), The political economy of hazards and disasters. Altamira Press, p. 241-264.

Chaouch N., Temimi M., Hagen S., Weishampel J., Medeiros S., Khanbilvardi R., 2012. A synergetic use of satellite imagery from SAR and optical sensors to improve coastal flood mapping in the Gulf of Mexico. Hydrological processes, vol. 26, n 11, p. 1617-1628.

Chaperon P., Danloux J., Ferry L.., 1993. Fleuves et rivières de Madagascar = Ony sy renirano eto Madagasikara. Paris, ORSTOM, $874 \mathrm{p}$.

Humanitarian OpenStreetMap Team, HOT sur Sambava [site web]. https://earth.esa.int/web/ sentinel/sentinel-technical-guides 
Institut National de la Statistique de Madagascar [site web]. https://www.instat.mg/rgph-3madagascar/historique/

Jumaux G., Quetelard H., Roy D., 2011. Atlas climatique de la Réunion. Météo-France, Direction interrégionale de la Réunion. $129 \mathrm{p}$.

Kiage L. M., Walker N. D., Balasubramanian S., Babin A., Barras, J., 2005. Applications of Radarsat1 synthetic aperture radar imagery to assess hurricane-related flooding of coastal Louisiana. International Journal of Remote Sensing, vol. 26, $\mathrm{n}^{\circ}$ 24, p. 5359-5380.

Mason D. C., Speck R., Devereux B., Schumann G. J. P., Neal J. C., Bates,P. D., 2010. Flood detection in urban areas using TerraSAR-X. IEEE Transactions on Geoscience and Remote Sensing, vol. $48, \mathrm{n}^{\circ} 2$, p. 882-894.

McFeeters S. K., 1996. The use of the Normalized Difference Water Index, NDWI) in the delineation of open water features. International journal of remote sensing, vol. $17, \mathrm{n}^{\circ} 7$, p. 1425-1432.

McMillan A., Morley J. G., Adams B. J., Chesworth S., 2006. Identifying optimal SAR imagery specifications for urban flood monitoring: a hurricane Katrina case study. In 4th international workshop on remote sensing for post-disaster response, Magdalene College, University of Cambridge, Cambridge, p. 25-26.

Rakotondrasoa F., 2016. Implementation of Disaster and Risk Management Policy in the East-Cost of Madagascar. JKAP, Jurnal Kebijakan dan Administrasi Publik), vol. 20, n 1, p. 84-94.

Satellites Sentinel-1 et 2 . Spécifications techniques (site web) https://earth.esa.int/web/ sentinel/sentinel-technical-guides

Wang Y., 2004. Using Landsat 7 TM data acquired days after a flood event to delineate the maximum flood extent on a coastal floodplain. International Journal of Remote Sensing, vol. $25, \mathrm{n}^{\circ} 5$, p. 959-974.

\section{ANNEXES}




\section{Annexe 1 - Niveaux de vigilance cyclonique fournis par la DGM (Direction Générale de la Météorologie) de Madagascar}

\begin{tabular}{|c|c|c|}
\hline $\begin{array}{c}\text { Niveau de } \\
\text { vigilance }\end{array}$ & Signification & Comportement \\
\hline $\begin{array}{c}\text { Verte ( } 5 \text { à } 2 \text { jours } \\
\text { avant la } \\
\text { catastrophe) }\end{array}$ & $\begin{array}{l}\text { CYCLONE: Mise en garde contre le risque } \\
\text { cyclonique; Une perturbation cyclonique évolue } \\
\text { dans la zone; elle présente un danger sans pour } \\
\text { autant qu'un délai puisse être indiqué de façon } \\
\text { précise pour les districts concernés. } \\
\text { HORS CYCLONE: Danger de fortes pluies; ou } \\
\text { vent très soutenu, ou risque de mer dangereuse }\end{array}$ & $\begin{array}{c}\text { Soyez attentifs - Avis } \\
\text { d'avertissement }\end{array}$ \\
\hline $\begin{array}{l}\text { Jaune ( } 48 \text { à } 24 \mathrm{~h} \\
\text { avant l'impact) }\end{array}$ & $\begin{array}{l}\text { CYCLONE: La menace se précise ; il y a danger } \\
\text { pour les districts concernés dans les jours qui } \\
\text { viennent. } \\
\text { HORS CYCLONE: Danger imminent de fortes } \\
\text { pluies; ou vent très soutenu, ou risque de mer } \\
\text { dangereuse. }\end{array}$ & Préparez-vous - Avis de menace \\
\hline $\begin{array}{c}\text { Rouge ( } 12 \mathrm{~h} \text { avant } \\
\text { l'impact) }\end{array}$ & $\begin{array}{l}\text { CYCLONE: Le cyclone menace à brève } \\
\text { échéance la localité et ses effets (coups de } \\
\text { vents forts, pluies diluviennes et marée de } \\
\text { tempête) constituent un danger pour la } \\
\text { population. } \\
\text { HORS CYCLONE: Danger de très fortes pluies; } \\
\text { ou inondations généralisées; ou glissements de } \\
\text { terrain; ou éboulements; ou mer } \\
\text { exceptionnellement dangereuse. }\end{array}$ & Confinez-vous - ne sortez pas \\
\hline $\begin{array}{c}\text { Bleue (un cyclone } \\
\text { tropical a traversé } \\
\text { le territoire) }\end{array}$ & $\begin{array}{l}\text { Fortes pluies, crues, fortes houles et vents } \\
\text { violents sont encorepossibles.Des résidus de } \\
\text { phénomènes, des dangers persistent } \\
\text { localement. }\end{array}$ & $\begin{array}{c}\text { Restez vigilants - Phase de } \\
\text { sauvegarde }\end{array}$ \\
\hline
\end{tabular}

source : http://www.primature.gov.mg/cpgu/wp-content/uploads/2018/08/MANUELSAP-.pdf 


\section{Annexe 2 - Niveaux d'alerte cyclonique fournis par le BNGRC (Bureau National de Gestion des Risques et des Catastrophes) de Madagascar}

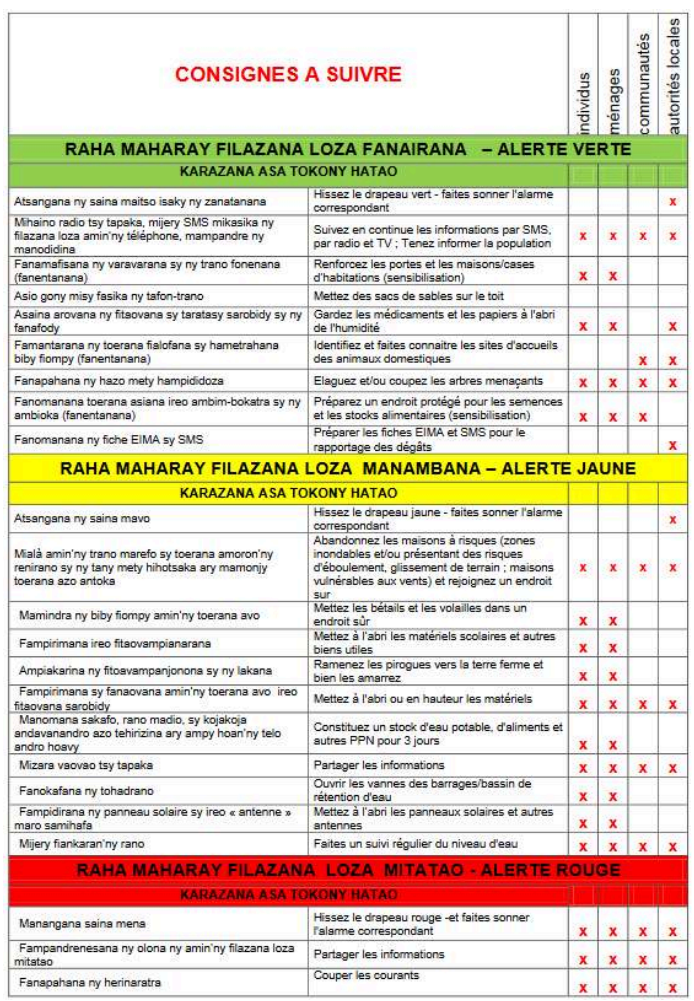

\section{RAHA MAHARAY FA FOANA NY FILAZANA LOZA - ALERTE BLEUE} KARAZANA ASA TOKONY HATAO

\begin{tabular}{|c|c|c|c|c|c|}
\hline Atsangana ny saina manga & $\begin{array}{l}\text { Hissez le drapegu bleu - faites sonner l'alarme } \\
\text { correspondant }\end{array}$ & & & $\mathbf{x}$ & $\mathbf{x}$ \\
\hline $\begin{array}{l}\text { Tokony ho malina hatrany ny mponina : rano fisotro } \\
\text { nampangotrahina, ny tariby mitondra herinaratra, ny } \\
\text { fiakaran'ny rano, ny fiotsahana'ny tany }\end{array}$ & $\begin{array}{l}\text { Maintenez létat de vigilance de la population: } \\
\text { eau potable, les fils électriques, la montée des } \\
\text { eaux, le glissement des terrains }\end{array}$ & $\mathbf{x}$ & $\mathbf{x}$ & $\mathbf{x}$ & $\mathbf{x}$ \\
\hline $\begin{array}{l}\text { Mampivory ny komity GRC ny amin'ny fanaovana } \\
\text { tombana sy ny famonjena isaky ny sektora }\end{array}$ & $\begin{array}{l}\text { Faites un rassemblement des membres de } \\
\text { comité GRC pour identifier les actions urgentes } \\
\text { à mener pour chaque secteur }\end{array}$ & & & & $\mathbf{x}$ \\
\hline Manao vonjy aina ho an'ny maratra sy marary & $\begin{array}{l}\text { Secours d'urgence pour les blessés et les } \\
\text { malades }\end{array}$ & $\mathbf{x}$ & $\mathbf{x}$ & $\mathbf{x}$ & $\mathbf{x}$ \\
\hline $\begin{array}{l}\text { Mamindra ny olona tavela any amin'ny toerana } \\
\text { dibodrano mankany amin'ny toerana voaharo }\end{array}$ & $\begin{array}{l}\text { Evacuation de la population dans les zones } \\
\text { inondées et à risque vers les sites } \\
\text { d'hébergement }\end{array}$ & & & $\mathbf{x}$ & $\mathbf{x}$ \\
\hline $\begin{array}{l}\text { fananganana ny lisitry ny traboina sy ny tsy } \\
\text { manankialofana }\end{array}$ & Établissement de la liste des sans abris & & & $\mathbf{x}$ & $\mathbf{x}$ \\
\hline $\begin{array}{l}\text { Fitantanana ny sites d'hebergement fanaovana } \\
\text { toerampivoahana, toerapidowana, fikarakarana ny rano } \\
\text { fisotro madio }\end{array}$ & Gestion des dons et des sites d'hébergement & $\mathbf{x}$ & $\mathbf{x}$ & $\mathbf{x}$ & $\mathbf{x}$ \\
\hline $\begin{array}{l}\text { Fanarahamaso ny trangan'aretina na valan'aretina } \\
\text { mety hiseho }\end{array}$ & Faites un suivi régulier du niveau d'eau & $\mathbf{x}$ & $\mathbf{x}$ & $\mathbf{x}$ & $\mathbf{x}$ \\
\hline $\begin{array}{l}\text { Manao ny tombana miaraka ny amin'ny fahavoazana } \\
\text { voalohany }\end{array}$ & Faites une évaluation rapide des dégâts & & & $\mathbf{x}$ & $\mathbf{x}$ \\
\hline $\begin{array}{l}\text { Mandefa ny tatitra voalohany any amin'ny BNGRC } \\
\text { amin'ny alalan'ny SMS, Radio BLU, antso antelefonina }\end{array}$ & $\begin{array}{l}\text { Envoyez les informations sur les impacts aux } \\
\text { autorités locales en utilisant le SMS, BLU et } \\
\text { appel téléphonique }\end{array}$ & $\mathbf{x}$ & $\mathbf{x}$ & $\mathbf{x}$ & $\mathbf{x}$ \\
\hline
\end{tabular}

Source : http://www.primature.gov.mg/cpgu/wp-content/uploads/2018/08/MANUELSAP-.pdf 


\section{NOTES}

1. Source FMI, WEO 2017.

2. Élaboration des normes contre l'inondation pour la construction des infrastructures routières

à Madagascar http://www.primature.gov.mg/cpgu/wp-content/uploads/2014/03/ NORMES\%20CONTRE\%20L_INONDATION\%20POUR\%20LA\%20CONSTRUCTION\%20DES\%20INFRASTRUCTURES\%20ROUTIERES.pdf

3. Source : Institut National de la Statistique de Madagascar. Ces chiffres sont des estimations, le dernier recensement officiel a eu lieu en 1993 (https://www.instat.mg/rgph-3-madagascar/ historique/).

4. https://tasks.hotosm.org/project/2651

\section{RÉSUMÉS}

Cette étude porte sur l'utilisation de l'imagerie satellite pour le suivi des cyclones et la caractérisation de leur impact. Deux événements récents sont étudiés à Madagascar: (i) Le cyclone Haruna, impactant la côte sud-ouest en février 2013, (ii) Le cyclone Enawo, touchant la côte nord-est en mars 2017. Le suivi de ces deux épisodes a montré la faisabilité de la mobilisation de l'imagerie satellitaire sur Madagascar pour une quantification des surfaces inondées associées aux cyclones. La combinaison des capteurs optiques et SAR (Synthetic Aperture Radar) à haute résolution temporelle et spatiale (HRS) abouti à : (i) un suivi en quasi temps réel d'Haruna par une surveillance des inondations pendant la crise et de la décrue en post-crise (ii) une cartographie des inondations en zone urbaine et dans les rizières de la région de Maroantsetra et Sambava, associées à Enawo, en combinant des images Sentinel-1 et 2, Pléiades et une démarche de cartographie participative.

This study focuses on the use of earth observation data for the monitoring of tropical cyclones and their impact in the South-western Indian Ocean. Two recent events that occurred in Madagascar are investigated: (i) Haruna, that hit the south-western coast in February 2013 and (ii) Enawo, that hit the north-eastern coast in March 2017. The results showed that satellite imagery is suitable for the quantification of hurricane-related floods over Madagascar territory. The synergetic use of optical and SAR (Synthetic Aperture Radar) sensors, with high temporal and spatial resolution allowed to : (i) carry out a near-real time monitoring of floods caused by Haruna during the crisis and a characterization of water level subsidence in post-crisis (combining SPOT 5, RADARSAT-2 and Pléiades images), (ii) a mapping of floods related to Enawo in urban areas and in rice fields in the regions of Sambava and Maroantsetra (combining Sentinel-1, Sentinel-2, and participatory mapping).

\section{INDEX}

Keywords : hurricane, Madagascar, Optical and SAR remote-sensing

Mots-clés : cyclone, Madagascar, télédétection optique, RADAR

Thèmes : Sur le Champ - Sur le Terrain 


\section{AUTEURS}

\section{THIBAULT CATRY}

Thibault Catry, catry@ird.fr, est ingénieur de recherche en traitement, analyse et représentation de l'information spatiale (télédétection) à l'IRD et membre de l'UMR ESPACE-DEV. Il a récemment publié:

- Pinel S., Bonnet M.-P., Da Silva S. et al., 2020. Flooding dynamics within an Amazonian floodplain: water circulation patterns and inundation duration. Water Resources Research [En ligne], vol. 56, n 1. https://doi.org/10.1029/2019WR026081

- Alexandre C., Johary R., Catry T. et al., 2020. A Sentinel-1 Based Processing Chain for Detection of Cyclonic Flood Impacts. Remote Sensing [En ligne], vol. 12, n 2. https://doi.org/10.3390/ rs12020252

- Catry T., Li Z., Roux E. et al., 2018. Wetlands and malaria in the Amazon: Guidelines for the use of synthetic aperture radar remote-sensing. International journal of environmental research and public health [En ligne], vol. 15, n³.10.3390/ijerph15030468

\section{CHRISTOPHE RÉVILLION}

Christophe Révillion, christophe.revillion@univ-reunion.fr, est ingénieur d'étude en traitement, analyse et représentation de l'information spatiale (télédétection) à l'Université de la Réunion et membre de l'UMR ESPACE-DEV. Il a récemment publié :

- Alexandre C., Rosa J., Catry T. et al., 2020. A Sentinel-1 Based Processing Chain for Detection of Cyclonic Flood Impacts. Remote Sensing [En ligne], vol. 12, n 2. https://doi.org/10.3390/ rs12020252

- Révillion C., Attoumane A., Herbreteau V., 2019. Homisland-IO: Homogeneous land use/land cover over the Small Islands of the Indian Ocean. Data [En ligne], vol. 4, $\mathrm{n}^{\circ} 2 . \mathrm{https}$ ://doi.org/ $10.3390 /$ data4020082

- Herbreteau V., Kassié D., Roux E. et al., 2018. Observer la Terre pour appréhender spatialement les inégalités de santé : regard historique et prospectif sur l'utilisation de la télédétection dans le domaine de la santé. Confins. Revue franco-brésilienne de Géographie/Revista franco-brasilera de Geografia [En ligne], n 37. https://doi.org/10.4000/confins.15362

\section{PASCAL MOUQUET}

Pascal Mouquet, pascal.mouquet@ird.fr, est ingénieur de recherche en production, traitement et analyse de données issues de la télédétection à l'IRD et membre de l'UMR ESPACE-DEV. Il a récemment publié :

- Alexandre C., Johary R., Catry T. et al., 2020. A Sentinel-1 Based Processing Chain for Detection of Cyclonic Flood Impacts. Remote Sensing [En ligne], vol. 12, n 2. https://doi.org/10.3390/ rs12020252

- Chambault P., Dalleau M., Nicet J.B., Mouquet P. et al., 2020. Contrasted habitats and individual plasticity drive the fine scale movements of juvenile green turtles in coastal ecosystems. Movement Ecology [En ligne], vol. 8, n 1, p.1-15. https://doi.org/10.1186/s40462-019-0184-2 - Bajjouk T., Mouquet P., Ropert M. et al., 2019. Detection of changes in shallow coral reefs status: Towards a spatial approach using hyperspectral and multispectral data. Ecological Indicators [En ligne], vol. 96, Part 1, p. 174-191. https://doi.org/10.1016/j.ecolind.2018.08.052.

\section{GWENAËLLE PENNOBER}

Gwenaëlle Pennober, gwenaelle.pennober@univ-reunion.fr, est professeure en géographie à l'Université de La Réunion (Chaire mixte IRD) et membre de l'UMR ESPACE-DEV. Elle a 
récemment publié :

- Alexandre C., Johary R., Catry T. et al., 2020. A Sentinel-1 Based Processing Chain for Detection of Cyclonic Flood Impacts. Remote Sensing [En ligne], vol. 12, n². https://doi.org/10.3390/ rs12020252

- Lemahieu A., Blaison A., Crochelet E. et al., 2017. Human-shark interactions: the case study of Reunion island in the south-west Indian Ocean. Ocean \& Coastal Management, $n^{\circ} 136$, p. 73-82. - Mahabot, M. M., Jaud, M., Pennober, G. et al., 2017. The basics for a permanent observatory of shoreline evolution in tropical environments; lessons from back-reef beaches in La Reunion Island. Comptes Rendus Geoscience, vol. 349, $\mathrm{n}^{\circ}$ 6-7, p. 330-340. 